\title{
Volume nucleation rates for homogeneous freezing in supercooled water microdroplets: results from a combined experimental and modelling approach
}

\author{
M. E. Earle ${ }^{1, *}$, T. Kuhn ${ }^{1, * *}$, A. F. Khalizov ${ }^{1, * * *}$, and J. J. Sloan ${ }^{1}$ \\ ${ }^{1}$ Department of Earth and Environmental Sciences, University of Waterloo, Waterloo, ON, Canada \\ *now at: Cloud Physics and Severe Weather Research Section, Environment Canada, Toronto, ON, Canada \\ ** now at: Department of Space Science, Luleå University of Technology, Kiruna, Sweden \\ ${ }^{* * * *}$ now at: Department of Atmospheric Sciences, Texas A\&M University, College Station, TX, USA
}

Received: 28 August 2009 - Published in Atmos. Chem. Phys. Discuss.: 28 October 2009

Revised: 31 May 2010 - Accepted: 12 August 2010 - Published: 27 August 2010

\begin{abstract}
Temperature-dependent volume nucleation rate coefficients for supercooled water droplets, $J_{V}(T)$, are derived from infrared extinction measurements in a cryogenic laminar aerosol flow tube using a microphysical model. The model inverts water and ice aerosol size distributions retrieved from experimental extinction spectra by considering the evolution of a measured initial droplet distribution via homogeneous nucleation and the exchange of vapour-phase water along a well-defined temperature profile. Experiment and model results are reported for supercooled water droplets with mean radii of 1.0, 1.7, and $2.9 \mu \mathrm{m}$. Values of mass accommodation coefficients for evaporation of water droplets and vapour deposition on ice particles are also determined from the model simulations. The coefficient for ice deposition was found to be $0.031 \pm 0.001$, while that for water evaporation was $0.054 \pm 0.012$. Results are considered in terms of the applicability of classical nucleation theory to the freezing of micrometre-sized droplets in cirrus clouds, with implications for the parameterization of homogeneous ice nucleation in numerical models.
\end{abstract}

\section{Introduction}

Cirrus clouds play a significant role in Earth's radiative balance due to their interactions with incident solar and surfaceemitted thermal radiation (Liou, 1986). The radiative in-

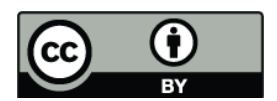

Correspondence to: J. J. Sloan

(sloanj@uwaterloo.ca) fluence of cirrus clouds is predicated on the concentrations, sizes, and shapes of the constituent ice particles (Lawson et al., 2006). Microphysical schemes in numerical models used to quantify and predict this influence require detailed parameterizations of the processes involved in ice initiation. Primary ice formation by homogeneous and/or heterogeneous nucleation provides an important starting point for describing the evolution of cloud properties due to mass-transfer evaporation/growth (the Bergeron-Findeisen process) and agglomeration/riming. Thus, ice nucleation processes in cirrus clouds continue to be an active area of atmospheric research.

At temperatures below about 235 to $237 \mathrm{~K}$, cirrus ice is formed primarily by homogeneous nucleation of aqueous droplets (Heymsfield et al., 2005). In the classical approach, homogeneous nucleation is considered to occur within the bulk volume of an aqueous droplet (Turnbull and Fisher, 1949), with a temperature-dependent rate coefficient, $J_{V}(T)$, having units of $\mathrm{cm}^{-3} \mathrm{~s}^{-1}$. Numerous laboratory studies have been conducted to determine atmospherically-relevant values of the nucleation rate coefficient. The bulk of this experimental work has focussed on the freezing of pure water droplets as a model system for ice formation in cirrus clouds and as a fundamental testing ground for the predictions of classical nucleation theory. Using water droplets suspended in air (DeMott and Rogers, 1990; Duft and Leisner, 2004; Krämer et al., 1999; Stöckel et al., 2005) and various oils (Butorin and Skripov, 1972; Taborek, 1985), values of $J_{V}(T)$ were determined from experimental measurements employing a range of droplet sizes and freezing detection methods. 
The experimentally-determined rate coefficients from the pure water studies are in general agreement with classical nucleation theory (Pruppacher, 1995). In some instances, however, there are large discrepancies among the results obtained from different studies; for example, there is a spread of over five orders of magnitude in values obtained at $240 \mathrm{~K}$ (Tabazadeh et al., 2002b). For some time, it has been unclear whether these large discrepancies were due solely to systematic differences in the experimental techniques and/or errors, or to some deficiency in the classical, volume-based approach for deriving nucleation rate coefficients from freezing data. The latter notion has been discussed recently, following the proposal that in some cases, crystal nucleation at the droplet surface is thermodynamically favoured over nucleation within the bulk volume (Djikaev et al., 2002; Tabazadeh et al., 2002a, b). The thermodynamic basis for surface nucleation is the potential decrease in surface energy when at least one of the crystal faces is in contact with the surrounding medium (air or oil in the relevant experimental studies), rather than the bulk liquid. The corresponding dependence on droplet surface area necessitates the introduction of a second, surface-specific rate coefficient, $J_{S}(T)$, with units of $\mathrm{cm}^{-2} \mathrm{~s}^{-1}$.

The total nucleation rate, $J_{\mathrm{t}}$, has units of $\mathrm{s}^{-1}$ and is the sum of contributions from volume- and surface-based processes:

$J_{\mathrm{t}}=J_{V} V+J_{S} \mathrm{~S}$

where $V$ and $S$ are the volume and surface area of the droplets, respectively. The relative contributions of each nucleation process depend on the nature of the interface with the droplet (air or oil) and droplet size (Tabazadeh et al., 2002b). The contribution of surface nucleation is expected to increase with decreasing particle size, because of the increasing surface-to-volume ratio. Duft and Leisner (2004) determined $J_{V}$ values for 19 and $49 \mu \mathrm{m}$ (radius) droplets levitated (in air) in an electrodynamic balance, and suggested that surface nucleation will only be important for particles with radii less than about $4 \mu \mathrm{m}$.

To assess further whether the classical, volume-based theory for homogeneous nucleation can be extended to micrometre-sized droplets suspended in air, additional laboratory studies are required. The evaporation of droplets in electrodynamic balances is prohibitive to the determination of $J_{V}(T)$ values for small droplets. Aerosol flow tubes (AFTs) and cloud chambers provide avenues for laboratory studies of micrometre-sized droplets; however, the determination of nucleation rate coefficients is complicated by the evaporation of liquid droplets and subsequent deposition on ice particles. The water vapour mass transfer growth of ice particles leads to increases in the (volume) ice fraction which are not directly related to the nucleation process. Previous studies of homogeneous ice nucleation in aerosol flow tubes have shown that at temperatures near the nucleation point, only a small fraction of the aerosols freeze ( 1 in $10^{4}$ to $\left.10^{6}\right)$, followed by significant vapour-phase exchange from the remaining liquid droplets to the nascent ice particles (Chelf and Martin, 2001).

To separate the contributions from homogenous nucleation and water vapour mass transfer in experimental measurements of aerosols in laminar flow tubes, microphysical models have been employed. Hung and Martin (2001) developed an inversion model in which the volume-based nucleation rate was treated as an adjustable parameter, and homogeneous nucleation and mass transfer were considered as sequential processes. The nucleation rate, $J_{V}(T)$, was varied iteratively to produce the best agreement between the volume fraction of ice calculated by the model, and that determined by Fourier-transform infrared (FTIR) observation, for a given freezing temperature.

The objective of this study is to combine experimental measurements from an aerosol flow tube with a microphysical model to determine $J_{V}(T)$ for micrometre-sized water droplets. The AFT apparatus has been characterized extensively using computational fluid dynamics (CFD) calculations (Khalizov et al., 2006b). Laminar flow conditions were demonstrated by quantifying thermal transfer in the flowing gas, thereby providing a time scale for kinetic measurements. The experimental conditions were designed to be appropriate for the application of the results to cirrus cloud formation. The microphysical model used in this study treats nucleation and mass transfer as simultaneous processes, thereby differing from the sequential approach used in previous models. This paper details the experimental setup and approach, as well as the design and implementation of the model. Values of $J_{V}(T)$ are reported from model simulations for pure water droplets with mean radii (maxima in the volume size distributions) of 1.0,1.7, and $2.9 \mu \mathrm{m}$. The results are considered with respect to the applicability of classical, volume-based, nucleation theory to the freezing of small droplets in cirrus clouds. The implications for bulk parameterizations of homogeneous nucleation in numerical models are also discussed.

\section{Experimental}

\subsection{Freezing experiments}

Freezing experiments were performed in a vertical aerosol flow tube (AFT), the details of which have been reported elsewhere (Khalizov et al., 2006a). The tube is equipped with copper cooling coils capable of operation down to $150 \mathrm{~K}$, and was designed to maintain laminar conditions with no temperature gradient-driven recirculation under very low flow velocities. It comprises four independently temperaturecontrolled copper sections, each $37.5 \mathrm{~cm}$ long, with an inner diameter (i.d.) of $8.9 \mathrm{~cm}$. Adjacent sections are joined by thin-walled stainless steel bellows, which minimize heat flow and allow individual sections to be maintained at different temperatures. The inner assembly (copper sections 
and bellows) is enclosed within an evacuated stainless steel jacket that provides thermal isolation from the laboratory environment. Chilled nitrogen coolant (mixture of liquid and gas) flows through coils affixed to the outer wall of each section. The wall temperature of each section is regulated using a LabVIEW® interface and feedback loop that controls a heater in the coolant gas flow. This system can maintain the average temperature of a section within $\pm 0.1 \mathrm{~K}$ of the set point value, with axial temperature deviations along the length of each section wall typically maintained within $\pm 0.5 \mathrm{~K}$. A set of cross-shaped copper fins is placed inside each section to transfer heat across the flow tube more efficiently, improving the cooling efficiency and minimizing radial temperature gradients in the gas flow.

A liquid water aerosol was generated by atomization or ultrasonic nebulization of Millipore®-filtered water, or by the heterogeneous condensation of humidified carrier gas on $\sim 20 \mathrm{~nm}$ dry sodium chloride $(\mathrm{NaCl})$ particles. In the latter case, the condensation nuclei were produced by atomizing a dilute, $10^{-4} \mathrm{~mol} \mathrm{~L}^{-1}(\mathrm{M})$ solution of reagent-grade $\mathrm{NaCl}$ (Sigma-Aldrich Co.) in Millipore®-filtered water, and passing the aqueous particles through a $60 \mathrm{~cm}$ long Nafion® diffusion dryer. The dry salt aerosol was pre-mixed with humidified nitrogen and introduced to the flow tube, where the salt particles deliquesced and continued to grow by water vapour uptake. The low $\mathrm{NaCl}$ concentration in the resulting droplets affected neither optical properties (DeMott and Rogers, 1990) nor freezing temperatures. We confirmed this in separate freezing experiments with $\mathrm{NaCl}$ solutions of several increasing concentrations. Changes in the optical and freezing properties became noticeable only for $\mathrm{NaCl}$ concentrations three orders of magnitude higher than those used here.

The experimental configuration is shown in Fig. 1. For each generation method, the droplets or nuclei were entrained in a dry nitrogen gas flow of 3 SLPM $\left(3 \mathrm{~L} \mathrm{~min}^{-1}\right.$ at standard conditions of $294 \mathrm{~K}$ and $1013 \mathrm{hPa}$ ). These externally-generated particles were introduced at the top of the cryogenic AFT via a central inlet (heated to prevent ice deposition), where they were then combined with a precooled flow of nitrogen carrier gas at 7 SLPM. When employing nebulization or atomization, dry nitrogen carrier gas was used. A mixture of dry (4 SLPM) and humidified (3 SLPM) nitrogen was used for the carrier flow when generating aerosol particles by heterogeneous condensation. The total flow rate was 10 SLPM in all cases.

The aerosol samples were exposed to well-defined temperature profiles as they flowed through the four tube sections, which will be designated A through D (top to bottom) in the following. Sections A and B were maintained at $240 \mathrm{~K}$ in all experiments to condition the aerosol. At the end of section B, the samples were composed solely of supercooled droplets. Tube sections C and D were then cooled to candidate freezing temperatures between 230 and $240 \mathrm{~K}$. At a total flow rate of 10 SLPM, the residence time of aerosol particles, from the

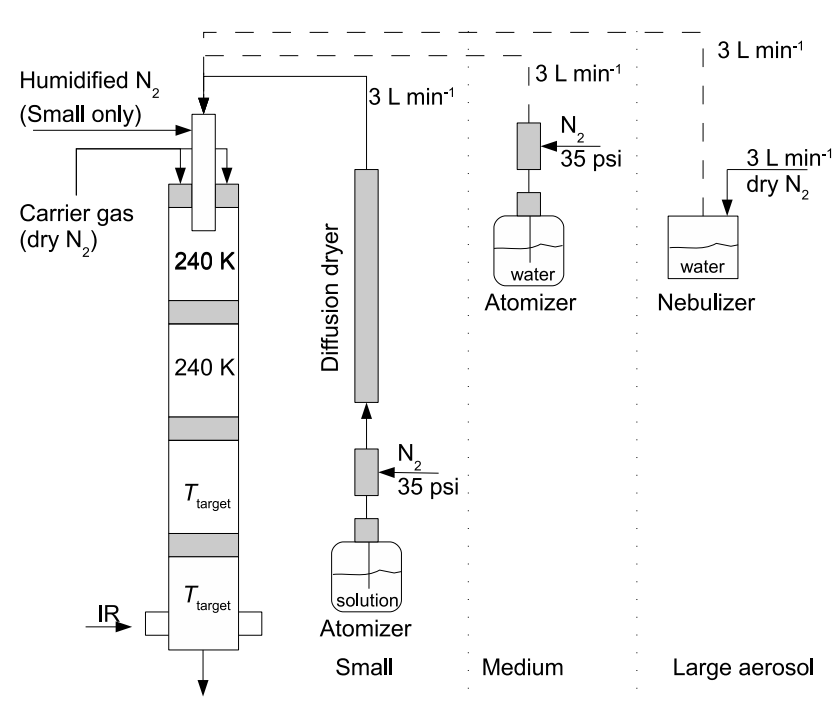

Fig. 1. Setup of cryogenic aerosol flow tube apparatus showing the three alternative configurations for aerosol generation.

point at which they are cooled following conditioning to the observation point (see Sect. 2.2), is about $35 \mathrm{~s}$.

\subsection{Infrared spectra and retrieval}

Infrared (IR) extinction spectra of the flowing aerosol were recorded in the lower half of section D. A collimated IR beam, modulated at $40 \mathrm{kHz}$ metrology frequency by a Michelson interferometer (Bruker Tensor 37), was passed through optical ports (capped with KRS-5 windows) on opposite sides of the flow tube, intersecting the aerosol. The transmitted IR beam was focussed by an off-axis parabolic mirror onto a mercury cadmium telluride (MCT) detector (FTIR-22-1.0, Infrared Associates). For each measurement, 80 scans were averaged over the frequency range from $450 \mathrm{~cm}^{-1}$ to $6000 \mathrm{~cm}^{-1}$, at $2 \mathrm{~cm}^{-1}$ resolution. Water vapour spectra recorded at $243 \mathrm{~K}$ were routinely subtracted from the measured extinction spectra to remove water vapour interference.

A remote sensing retrieval procedure developed in our laboratory was used to determine the phase composition and corresponding size distribution(s) of the supercooled water and/or ice particles from their IR spectra (Zasetsky et al., 2004a, 2007). The retrieval procedure is based on a minimization of the variance between the measured spectrum and a calculated spectrum that is the linear combination of a set of monodisperse reference spectra. This basis set of reference spectra is calculated for 96 radii that are equally spaced on a logarithmic size scale in the range between 0.05 and $11.8 \mu \mathrm{m}$. Spectral basis sets for both supercooled water and ice are calculated using Mie scattering code (Bohren and Huffman, 1983), with frequency-dependent complex indices of refraction (Zasetsky et al., 2005). This approach assumes sphericity, which is a reasonable approximation, because the average 
particle radii are in the $1 \mu \mathrm{m}$ to $5 \mu \mathrm{m}$ range and images made with an optical microscope (Zasetsky et al., 2007) mounted temporarily in the aerosol flow (in tube section D) show that under the conditions of these experiments, the particles have near-unity aspect ratios.

The retrieval procedure determines a particle size distribution using a nonlinear least-squares algorithm. The resulting size distribution is the number concentration of particles in each of the 96 size bins (corresponding to the radii in the basis set) which produce the calculated spectrum that best fits the measured spectrum. Ice and/or supercooled water particle distributions are obtained simultaneously in this way. Number size distributions can be converted to volume size distributions by multiplying the number concentration of particles in each bin by $(4 / 3) \pi r^{3}$, where $r$ is the bin radius. Volume size distributions are used in all analyses because they are directly related to mass, which is conserved in kinetic processes. Comparison of size distributions retrieved from IR spectra and scanning electron microscope (SEM) images of commercial $\mathrm{SiO}_{2}$ microspheres indicated that the retrieval procedure is accurate to better than $\pm 5 \%$ (Zasetsky et al., 2007).

\subsection{Initial aerosol size distributions}

To estimate the sizes of the conditioned aerosols from each generation method, experiments were conducted with all four flow tube sections held at the conditioning temperature of $240 \mathrm{~K}$. Excess water vapour was removed by freezing onto the wall and fins upon cooling in the first two sections, and the particles did not experience appreciable changes in size beyond this point. Analysis of the resulting extinction spectra using the characterization procedure (detailed in Sect. 2.2) produced the volume size distributions of supercooled water droplets for each generation method shown in Fig. 2. The conditioned aerosols produced by heterogeneous condensation have radii between approximately 0.4 and $2 \mu \mathrm{m}$, with a maximum in the volume distribution at about $1 \mu \mathrm{m}$. Those produced by the constant output atomizer have radii between 0.6 and $5 \mu \mathrm{m}$, with a mean of approximately $1.7 \mu \mathrm{m}$. Nebulization produces conditioned aerosols with radii between 1 and $7 \mu \mathrm{m}$, with a mean of about $2.9 \mu \mathrm{m}$. Based on these sizes, the aerosols produced by condensation, atomization, and nebulization will henceforth be referred to as small, medium, and large droplets, respectively. Volume concentrations in each of the 96 size bins considered by the retrieval procedure are indicated in Fig. 2. These concentrations define the range of typical values in freezing experiments for each droplet size, which are considered further in Sect. 4.

\subsection{Axial temperature profiles}

The axial temperature profile experienced by the aerosol cannot be inferred solely from measurements made at the tube wall and/or at fixed radial positions in the flow (e.g. in the

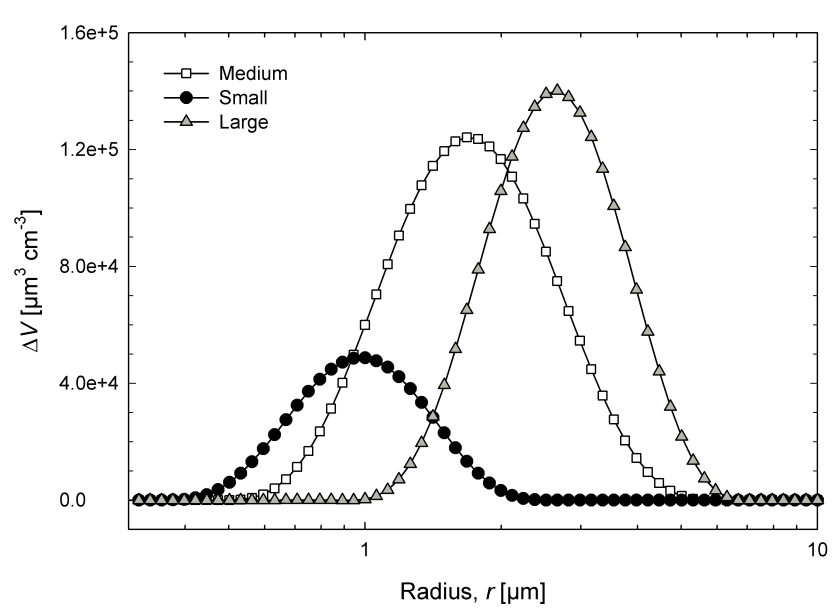

Fig. 2. Volume size distributions determined from the IR retrieval procedure (Sect. 2.2) for supercooled water droplets after conditioning at $240 \mathrm{~K}$. The droplets have mean radii of $1.0 \mu \mathrm{m}$ (small), $1.7 \mu \mathrm{m}$ (medium), and $2.9 \mu \mathrm{m}$ (large), corresponding to the generation methods of heterogeneous condensation, atomization, and nebulization, respectively (Fig. 1).

centre), because the aerosol is not distributed evenly over the cross-section of the flow. Moreover, even at the relatively low linear flow velocities of our experiments (a few $\mathrm{cm}$ per second), small residual radial temperature gradients may remain (typically less than $0.5 \mathrm{~K}$ in the region of minimum temperature, where most freezing occurs; see Sect. 4.3). To account for these factors, CFD calculations were used to determine the cross-sectional- and mass-averaged temperature profiles in the AFT (Khalizov et al., 2006a). It has been shown that CFD computations accurately represent the conditions to which the aerosol particles are exposed as they travel through the AFT, including not only the temperature distributions, but also important flow characteristics such as gas streamlines and particle tracks. These results show that the particle motion is linear and follows the gas flow, which is laminar under the conditions of our experiments. Laminar flow is necessary both to avoid agglomeration and to ensure that the timescales used in the subsequent kinetic analyses are correct.

CFD profiles were calculated for each candidate freezing temperature. Using the calculated aerosol flow velocities, which are about $3 \mathrm{~cm} \mathrm{~s}^{-1}$ for a total flow rate of 10 SLPM, the temperature profiles as a function of the axial coordinate were converted into profiles as a function of aerosol residence time. The latter profiles are required by the kinetic model to interpret the aerosol transformations during freezing. This model is detailed in the following section.

\section{Model description}

Implemented in Matlab (The Mathworks, Inc.), the model uses the measured volume distributions of supercooled water 
and ice aerosol particles obtained from freezing experiments to determine temperature-dependent homogeneous nucleation rate coefficients. The distributions measured at different temperatures represent the aerosol particles at the various stages of the freezing event. Before freezing, the initial distribution is that of a pure (supercooled) water aerosol that has been conditioned with all wall temperatures set to $240 \mathrm{~K}$ (Fig. 2). The final supercooled water and/or ice distributions are taken from experiments conducted with the bottom two tube sections $\mathrm{C}$ and $\mathrm{D}$ at a given candidate freezing temperature.

The model has two components: a microphysical simulation and a minimization step. The microphysical component consists of a series of rate processes describing homogeneous ice nucleation and (diffusion-limited) exchange between aerosol particles and water vapour. The exchange accounts for mass transfer between the liquid and frozen particles via evaporation, diffusion, and condensation/deposition. All processes use temperature-dependent rate constants and include the influence of the Kelvin effect. Nucleation and vapour-phase exchange processes are considered simultaneously, as opposed to the sequential approach used previously in similar models (Chelf and Martin, 2001; Hung et al., 2002; Hung and Martin, 2001). Beginning with the initial supercooled water aerosol distribution, the microphysical component solves the time-dependent differential equations describing the relevant rate processes, subject to the axial AFT temperature profile and the nucleation rate function $J_{V}(T)$. The calculations produce time-dependent volume distributions of supercooled water and/or ice. The normalized sum of squares of differences, $\chi$, between the calculated and measured (in AFT section D) supercooled water and ice distributions (see Eq. 16) is minimized by varying the function $J_{V}(T)$.

\subsection{Microphysical simulation}

The volume distributions of supercooled water and ice output by the retrieval procedure (Sect. 2.2) give the volume concentration of particles in each of the 96 size bins corresponding to the radii specified in the spectral basis set. These radii will be referred to as nodes in the following. Particles in the size bins are assumed to reside only at the nodes.

The rate of increase in the number of ice particles in node $i, N_{i}^{s}$, due to homogeneous nucleation is given by the following:

$d N_{i}^{s} / d t=N_{i}^{l} J_{V}(T) v_{i}$

where $N_{i}^{l}$ is the number concentration of liquid droplets in node $i$, and $v_{i}=(4 / 3) \pi\left(r_{i}^{l}\right)^{3}$ is the volume of a liquid droplet in node $i$ having radius $r_{i}^{l}$. The decrease in the number of water droplets resulting from homogeneous nucleation is determined from the increase in the number of ice particles:

$-d N_{i}^{l} / d t=d N_{i}^{s} / d t$
Equations (2) and (3) are based on the assumptions that nucleation events in droplets are independent, that ice formation is the result of only one nucleation event per droplet, and that once nucleated, the entire droplet is frozen instantaneously.

The temperature dependence of the nucleation rate is given by classical nucleation theory (Turnbull and Fisher, 1949):

$J_{V}(T)=N_{V} \frac{k T}{h} \exp \left(-\frac{\Delta F_{V}}{k T}\right)$

where $N_{V}=3.35 \times 10^{22} \mathrm{~cm}^{-3}$ is the number concentration of water molecules in the droplet volume, $k$ is the Boltzmann constant, and $h$ is the Planck constant. The term $\Delta F_{V}$ represents the sum of the free energy of nucleus formation in the bulk volume and the activation energy for the diffusion of water molecules across the liquid-solid boundary of the nucleus (Pruppacher and Klett, 1998; Turnbull and Fisher, 1949). The temperature dependence of $J_{V}$ is given by the strong temperature dependence of the exponential factor, as well as that of the pre-factor and $\Delta F_{V}$. The latter was modelled using the expression $\Delta F_{V}=A_{V}-B_{V} T$. Incorporating the parameters $A_{V}$ (in units of energy) and $B_{V}$ (in units of energy divided by temperature) into the formulation of $J_{V}(T)$ used in the model (Eq. 4) gives:

$J_{V}(T)=N_{V} \frac{k T}{h} \exp \left(-\frac{A_{V}-B_{V} T}{k T}\right)$

The temperature dependence in Eq. (5) governs the number of ice particles formed as the temperature of the supercooled water aerosol changes subject to the temperature profile in the flow tube. Minimization of the function $\chi$ by varying the parameters $A_{V}$ and $B_{V}$ produces the temperature-dependent nucleation rates, $J_{V}(T)$, that are the desired output.

Diffusion-limited exchange processes result from differences between the saturation vapour pressures above particles and the partial pressure of water vapour in the surrounding gas. These processes include the evaporation and condensation growth of the liquid particles and the deposition growth of the ice particles. The rate of radius change for water droplets and ice particles by the combination of these processes is determined as follows (Hinds, 1999):

$$
\begin{aligned}
& \frac{d r_{i}^{l}}{d t}=\frac{D_{v}^{*}\left(r_{i}^{l}\right) M_{\mathrm{w}}}{R \rho_{\mathrm{w}}} \frac{1}{r_{i}^{l}}\left(\frac{p_{\infty}}{T_{\infty}}-\frac{p_{i}}{T_{i}^{l}}\right) \\
& \frac{d r_{i}^{s}}{d t}=\frac{D_{v}^{*}\left(r_{i}^{s}\right) M_{\mathrm{w}}}{R \rho_{\text {ice }}} \frac{1}{r_{i}^{s}}\left(\frac{p_{\infty}}{T_{\infty}}-\frac{p_{\text {ice }}}{T_{i}^{s}}\right)
\end{aligned}
$$

where $r_{i}^{l}$ is the radius of liquid droplets in node $i ; r_{i}^{s}$ is the radius of ice particles in node $i ; D_{v}^{*}\left(r_{i}^{l}\right)$ and $D_{v}^{*}\left(r_{i}^{s}\right)$ are the diffusion coefficients of water vapour near liquid and frozen aerosol particles; $M_{\mathrm{w}}$ is the molar mass of water; $R$ is the ideal gas constant; $\rho_{\mathrm{W}}$ is the density of supercooled water; $\rho_{\text {ice }}$ is the density of ice; $p_{\infty}$ is the far-field water partial pressure; $p_{i}$ is the saturation vapour pressure above droplets 
in node $i$ (corrected for the Kelvin effect); $p_{\text {ice }}$ is the saturation vapour pressure above ice; $T_{\infty}$ is the ambient gas temperature at locations far removed from the droplets; $T_{i}^{l}$ and $T_{i}^{s}$ are the surface temperatures of the water droplets and ice particles, respectively. It is assumed that $T_{\infty}=T_{i}^{l}=T_{i}^{s}$, because the gain or release of heat by particles during condensation/deposition or evaporation will not change the particle surface temperatures by more than $0.01 \mathrm{~K}$ (Chelf and Martin, 2001). The suitability of this approximation for freezing experiments in the present study is considered further in Sect. 4.3.

The dependence of the gas-phase diffusion coefficients, $D_{v}^{*}\left(r_{i}^{l}\right)$ and $D_{v}^{*}\left(r_{i}^{s}\right)$, on the radii of water and ice particles follows from the approach of Fuchs, which corrects for discontinuities in the vapour density above the particles when their radii approach the mean free path, $\lambda$, of vapour molecules (Pruppacher and Klett, 1998):

$$
D_{v}^{*}\left(r_{i}^{l / s}\right)=\frac{D_{v}}{\left[\frac{r_{i}^{l / s}}{r_{i}^{l / s}+\Delta v}+\frac{D_{v}}{r_{i}^{l / s} \alpha_{\mathrm{w} / \mathrm{ice}}}\left(\frac{2 \pi M_{\mathrm{w}}}{R T_{i}^{l / s}}\right)^{2}\right]}
$$

In the above expression, $D_{v}$ is the vapour diffusivity of water in air, $\Delta v$ is the distance of the vapour continuum above the particle surface (taken as $1.3 \lambda$ in the present study), and $\alpha_{\mathrm{w}}$ and $\alpha_{\text {ice }}$ are the mass accommodation coefficients for the evaporation of liquid droplets (evaporation coefficient) and vapour deposition on ice particles (deposition coefficient), respectively.

The values of $\alpha_{\mathrm{w}}$ and $\alpha_{\text {ice }}$ required for computation of the corrected diffusion coefficients $D_{v}^{*}\left(r_{i}^{l}\right)$ and $D_{v}^{*}\left(r_{i}^{s}\right)$ are not well known. Under temperature conditions characteristic of our freezing experiments, previously reported values of $\alpha_{\mathrm{w}}$ vary from 0.04 to approximately 0.5 (Li et al., 2001; Shaw and Lamb, 1999), while those of $\alpha_{\text {ice }}$ span three orders of magnitude from approximately 0.001 to 0.6 (Choularton and Latham, 1977; Haynes et al., 1992; Magee et al., 2006; Pratte et al., 2006). Therefore, $\alpha_{\mathrm{w}}$ and $\alpha_{\mathrm{ice}}$ are treated as adjustable parameters in the model, and are iteratively varied during the minimization procedure (Sect. 3.2).

The far-field water partial pressure, $p_{\infty}$, is calculated from the total concentration of gaseous water molecules (vapour), $N_{\mathrm{v}}$, using the ideal gas law:

$p_{\infty}=N_{\mathrm{v}} k T_{\infty}$

The value of $N_{\mathrm{v}}$ is determined by assuming that the concentration of gaseous water is in a steady-state with respect to condensation and evaporation to and from liquid droplets and deposition on ice particles, and is subject to losses to the icecoated tube walls and fins.

$$
\begin{aligned}
& \frac{d N_{\mathrm{v}}}{d t}=-\sum_{i=1}^{96}\left(\frac{1}{v_{\mathrm{w}} \rho_{\mathrm{w}}} \frac{d m_{i}^{l}}{d t}+\frac{1}{v_{\text {ice }} \rho_{\text {ice }}} \frac{d m_{i}^{s}}{d t}\right) \\
& -k_{\text {loss }}\left(N_{\mathrm{v}}-N_{\mathrm{v}}^{\text {ice }}\right)
\end{aligned}
$$

The two terms in the summation represent the contributions from the liquid droplets and ice particles, where $m_{i}^{l}$ is the mass concentration of liquid droplets in node $i, m_{i}^{s}$ is the mass concentration of ice particles in node $i$, and $v_{\mathrm{w}}$ and $v_{\text {ice }}$ are the molecular volumes of liquid water and ice, respectively. The rates of change of mass concentration for liquid and ice particles in node $i$ due to mass transfer are given by:

$$
\begin{aligned}
\frac{d m_{i}^{l}}{d t} & =4 \pi r_{i}^{l 2} \rho_{\mathrm{w}} N_{i}^{l} \frac{d r_{i}^{l}}{d t} \\
\frac{d m_{i}^{s}}{d t} & =4 \pi r_{i}^{s 2} \rho_{\mathrm{ice}} N_{i}^{s} \frac{d r_{i}^{s}}{d t}
\end{aligned}
$$

The final term in Eq. (10) represents the loss of gaseous water to the ice-coated tube walls and fins, where $N_{\mathrm{v}}^{\text {ice }}$ is the concentration of vapour molecules given by the saturation vapour pressure of ice, and $k_{\text {loss }}$ is the first-order rate constant for radial diffusion to the walls in a laminar flow (Houzelot and Villermaux, 1977; Villermaux, 1971):

$k_{\text {loss }}=\frac{\mu D_{v}}{r_{\mathrm{t}}^{2}}$

In Eq. (13), $\mu=3.66$ is the Sherwood number for a cylindrical pipe, and $r_{\mathrm{t}}$ is the tube radius. In the present study, $k_{\text {loss }}=0.138 \mathrm{~s}^{-1}$ at $240 \mathrm{~K}$ (Khalizov et al., 2006b).

The wall- and fin-loss term in Eq. (10) is generally much smaller (by a factor of 10 to 20) than the sum of the contributions from droplets and ice particles. Frost formation on the tube wall and fins could lead to an increase in the Sherwood number, and corresponding increases in vapour losses; however, visual inspection of the lower tube sections showed no signs of frost. Considering the negligible absolute amount of ice on the walls and the suppression of convective mass transfer under the conditions of our experiments (Khalizov et al., 2006a, b), we do not expect any variability in the vapour losses due to frost.

From Eqs. (6) and (7), the changes in size of liquid water droplets and ice particles are determined by the difference between the far-field vapour pressure and the saturation vapour pressures of particles. The number of particles in a node may change due to size changes of particles resulting from condensation/deposition growth and evaporation. This exchange among nodes was implemented in the model using the approach of Prakash et al. (2003), in which the change in the number of liquid droplets in each node $i$ is considered in terms of four vapour pressure scenarios:

$$
\begin{aligned}
& \frac{d N_{i}^{l}}{d t}= \\
& \left\{\begin{array}{lll}
\frac{1}{\left(v_{i}-v_{i-1}\right) \rho_{\mathrm{w}}} \frac{d m_{i-1}^{l}}{d t} & \text { if } p_{\infty}>p_{i-1} & \text { (growthin bin } i-1) \\
-\frac{1}{\left(v_{i+1}-v_{i}\right) \rho_{\mathrm{w}}} \frac{d m_{i+1}^{l}}{d t} & \text { if } p_{\infty}<p_{i+1} & \text { (evaporation in bin } i+1) \\
-\frac{1}{\left(v_{i+1}-v_{i}\right) \rho_{\mathrm{w}}} \frac{d m_{i}^{l}}{d t} & \text { if } p_{\infty}>p_{i} & \text { (growthin bin } i) \\
\frac{1}{\left(v_{i}-v_{i-1}\right) \rho_{\mathrm{w}}} \frac{d m_{i}^{l}}{d t} & \text { if } p_{\infty}<p_{i} & \text { (evaporationin } \operatorname{bin} i)
\end{array}\right.
\end{aligned}
$$


where $v_{i}$ is the volume of a particle in node $i$, and $v_{i-1}$ and $v_{i+1}$ are the volumes of particles in adjacent size bins. The first two terms in Eq. (14) will increase the number density of particles in node $i$, while the last two terms will decrease the number density. An equivalent set of equations is used to describe the change in the number of ice particles in each node $i$ :

$$
\begin{aligned}
& \frac{d N_{i}^{s}}{d t}= \\
& \left\{\begin{array}{l}
\frac{1}{\left(v_{i}^{s}-v_{i-1}^{s}\right) \rho_{\text {ice }}} \frac{d m_{i-1}^{s}}{d t}-\frac{1}{\left(v_{i+1}^{s}-v_{i}^{s}\right) \rho_{\text {ice }}} \frac{d m_{i}^{s}}{d t} \\
\text { if } p_{\infty}>p_{\text {ice }} \quad \text { (growth) } \\
-\frac{1}{\left(v_{i+1}^{s}-v_{i}^{s}\right) \rho_{\text {ice }}} \frac{d m_{i+1}^{s}}{d t}+\frac{1}{\left(v_{i}^{s}-v_{i-1}^{s}\right) \rho_{\text {ice }}} \frac{d m_{i}^{s}}{d t} \\
\text { if } p_{\infty}<p_{\text {ice }} \quad \text { (evaporation) }
\end{array}\right.
\end{aligned}
$$

There are only two possibilities in this case, because $p_{\text {ice }}$ is a function of temperature only, and does not vary for adjacent nodes.

The initial inputs for the microphysics component are the initial volume distribution of supercooled droplets, the time evolution of temperature (determined from the flow velocity and axial temperature profile, as described in Sect. 2.4), the first guesses of values of $A_{V}$ and $B_{V}$ for the calculation of the nucleation rate (Eq. 5), and the mass accommodation coefficients $\alpha_{\mathrm{w}}$ and $\alpha_{\text {ice }}$ for the calculation of mass transfer by evaporation/growth. By performing the steps implied by Eqs. (2) to (15), the model calculates the time evolution of the far-field partial pressure, the number fraction of droplets frozen (ratio of number of droplets frozen to initial number of droplets), and thereby the evolution of the supercooled water and ice distributions.

\subsection{Minimization}

The minimization procedure iteratively varies the values of $A_{V}, B_{V}, \alpha_{\mathrm{w}}$ and $\alpha_{\text {ice }}$ to minimize the normalized sum of squares of differences, $\chi$ (Eq. 16 below), between the final volume distributions of supercooled water and ice aerosol particles measured in freezing experiments and those calculated by the model.

$$
\chi=\frac{\sum_{i=1}^{96}\left(V_{i}^{l, \exp }-V_{i}^{l, \text { calc }}\right)^{2}+\sum_{i=1}^{96}\left(V_{i}^{s, \exp }-V_{i}^{s, \text { calc }}\right)^{2}}{\left(\sum_{i=1}^{96}\left(V_{i}^{l, \exp }+V_{i}^{s, \exp }\right)\right)^{2}}
$$

In the above equation, $V_{i}^{l}$ and $V_{i}^{s}$ are the volume densities of liquid droplets and ice particles, respectively, in node $i$. The additional superscripts indicate whether volume densities are from experimental (exp) or calculated (calc) distri- butions. The model can be run for a single freezing experiment at a given candidate freezing temperature, or for several freezing experiments, performed at different candidate freezing temperatures, simultaneously. In the latter case, $\chi$ is evaluated separately for each experiment using Eq. (16), and then averaged over all of the experiments. In either case, the model iteratively varies a single set of $A_{V}, B_{V}, \alpha_{\mathrm{w}}$, and $\alpha_{\text {ice }}$ values for all of the constituent experiments to minimize the final (averaged) value of $\chi$.

The minimization uses the fminsearch minimizer from the Matlab standard library, which finds a minimum for an unconstrained multivariable function using the simplex search method (Lagarias et al., 1998). The strong temperature dependence of homogeneous nucleation rates complicates the minimization, as $A_{V}$ and $B_{V}$ pairs producing reasonable values of $J_{V}$ for a given temperature form a narrow strip on a plot of $A_{V}$ vs. $B_{V}$. Lines of constant $J_{V}$ are given by $A_{V}=B_{V} T-C$, where $C$ is a constant. If one changes $A_{V}$ and $B_{V}$ by moving along such a line, $J_{V}$ does not change (for a given temperature), but its temperature dependence does. If, on the other hand, one moves perpendicular to such a line, $J_{V}$ changes exponentially, while its temperature dependence changes little. This situation necessitates the use of extremely small steps in the minimization to maintain reasonable values of $J_{V}$, which slows down the calculation process dramatically.

To circumvent this issue, the parameters $A_{V}$ and $B_{V}$ were transformed to new parameters $a_{V}$ and $b_{V}$, which decouple the value of $J_{V}$ from its temperature dependence, greatly enhancing the performance of the minimizer. The parameters $a_{V}$ and $b_{V}$ are the inclination and offset when $J_{V}$ is approximated by a straight line on a $\log J_{V}$ vs. $T$ plot:

$\log \frac{J_{V}}{J_{0}}=a_{V}\left(T-T_{0}\right)+b_{V}$

where $J_{0}=1 \mathrm{~cm}^{-3} \mathrm{~s}^{-1}$ provides the correct units. Changing $a_{V}$ or $b_{V}$ is equivalent to moving parallel or perpendicular, respectively, to the lines of constant $J_{V}$ on the $A_{V}$ vs. $B_{V}$ plot. The coordinate transformation is accomplished by developing $\log J_{V}(T)$ in a Taylor series expansion up to the first order, around a temperature $T_{0}$ within the typical freezing range for our experiments:

$a_{V}=\frac{1}{T_{0} \ln 10}\left(1+\frac{A_{V}}{k T_{0}}\right)$

$b_{V}=\frac{1}{\ln 10}\left(-\frac{A_{V}-B_{V} T_{0}}{k T_{0}}+\ln \frac{N_{V} k T_{0}}{h J_{0}}\right)$

For these experiments, a value of $T_{0}=236.15 \mathrm{~K}$ was used. The new parameters $a_{V}$ and $b_{V}$ are used only in the minimization component of the model. In the microphysics component, $a_{V}$ and $b_{V}$ are transformed back to $A_{V}$ and $B_{V}$, which are used to calculate $J_{V}$ for each iteration. 


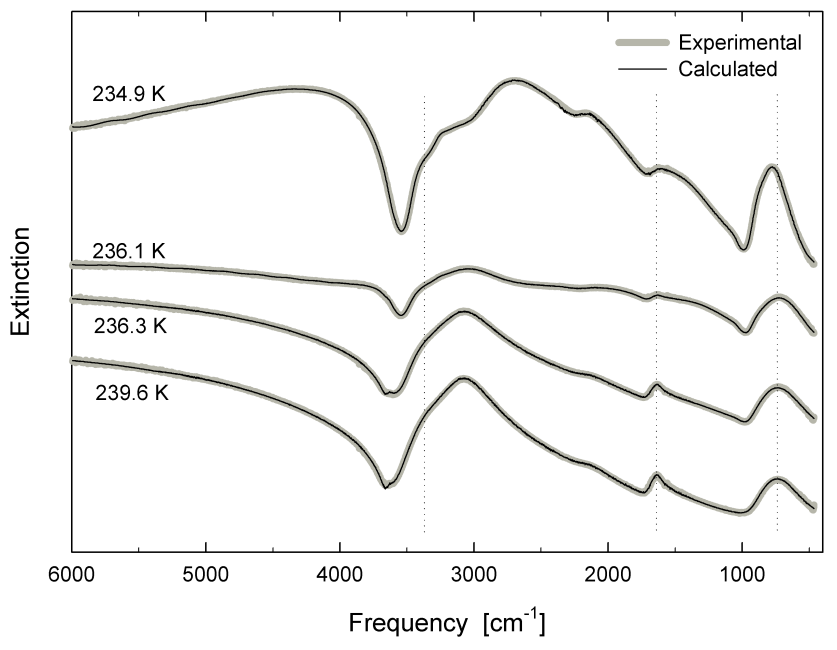

Fig. 3. Experimental extinction spectra obtained from freezing experiments using medium droplets generated by atomization at different candidate freezing temperatures. The spectra calculated from the retrieved size distributions are overlaid in black.

\section{Results and discussion}

\subsection{Changes in infrared spectra and aerosol size distributions upon cooling}

Extinction spectra were recorded for pure water aerosol as a function of the candidate freezing temperature. The spectra obtained at $239.6,236.3,236.1$, and $234.9 \mathrm{~K}$ are shown in Fig. 3 for the representative case of medium droplets produced by atomization. The freezing onset (homogeneous nucleation point) is indicated by distinct changes in the spectra between 236.3 and $236.1 \mathrm{~K}$; specifically, broadening of the $\mathrm{O}-\mathrm{H}$ vibrational stretching feature at $\sim 3000 \mathrm{~cm}^{-1}$ and the $\mathrm{H}-\mathrm{O}-\mathrm{H}$ bending feature at $\sim 1640 \mathrm{~cm}^{-1}$. These changes become more pronounced when the temperature is reduced to $234.9 \mathrm{~K}$ and a larger number of droplets are frozen, with the $\mathrm{O}-\mathrm{H}$ stretching feature exhibiting a marked change in shape. In addition, the $\mathrm{H}$-bonding (librational) feature at $\sim 700 \mathrm{~cm}^{-1}$ sharpens and shifts to higher wavenumbers.

The above changes in absorption features associated with freezing are all in accordance with previous reports (Cziczo and Abbatt, 1999; Clapp et al., 1995). Small variations in these spectral features at warmer temperatures are also evident from comparison of the spectra at 239.6 and $236.3 \mathrm{~K}$. In general, the spectral features that are characteristic of ice gradually become stronger as the temperature is decreased from near $273 \mathrm{~K}$ toward the nucleation point. A previous study in our laboratory (Zasetsky et al., 2004b) attributed these changes to the formation of low-density domains, consisting of ice-like clusters of water molecules, within the supercooled droplets.

The changes in extinction that occur above about $4000 \mathrm{~cm}^{-1}$, where neither water nor ice absorb, are due to variations in scattering. After ice nucleation has occurred in a small fraction of supercooled droplets, the changes in slope in this region are due primarily to the growth of nascent ice particles by vapour diffusion from the remaining liquid droplets. The growth process can be characterised by retrieving temperature-dependent size distributions of supercooled water and/or ice aerosol particles from experimental spectra using the procedure outlined in Sect. 2.2.

Retrieved volume size distributions of hydrometeors are shown in Fig. 4 for the same set of freezing experiments using medium droplets. The numerical values of the volume concentrations are omitted in these and subsequent size distribution plots for clarity (see Fig. 2 for typical values for experiments using each initial droplet size). The spectra calculated from the retrieved size distributions are overlaid on the experimental spectra in Fig. 3.

Figure 4 shows that the sample at $239.6 \mathrm{~K}$ was composed entirely of supercooled water droplets, with a mean radius of $\sim 1.7 \mu \mathrm{m}$. The apparent contribution from ice particles with radius approximately equal to that of the water droplets arises because the refractive indices, and therefore the spectra, of supercooled water and ice are very similar, making it difficult to distinguish between them by optical spectroscopy. As a result, experimental noise in the measured spectra can introduce small uncertainties in the retrieved ice and water distributions. This was discussed extensively by Zasetsky et al. (2004a). Similar small ice features are observed in the distributions at $236.1 \mathrm{~K}$ and $234.9 \mathrm{~K}$, as well as at warmer temperatures (not shown). These features cannot be eliminated (unless the measured spectra are completely noise free), and since they represent a negligible fraction of the total volume (see below), they were removed from the distributions prior to applying the microphysics model.

The secondary liquid water and ice features present at larger radii in Fig. 4 (e.g. the water peaks in the vicinity of $10 \mu \mathrm{m}$ at $239.6 \mathrm{~K}$ and $236.1 \mathrm{~K}$ and the "tails" on the water and ice distributions at the largest radii) cannot result from liquid droplets having large radii, because such large droplets would freeze rapidly under the conditions of our experiments. Our previous experience suggests that these apparent features are probably caused by slight shifts in the spectrometer baseline (less than $0.1 \%$ ), which cause contributions to the spectra that have the same appearance as spectra of very large particles. It has been shown previously that these baseline shifts do not affect the results for smaller radii in the size range of the present experiments (Zasetsky et al., 2004a). Thus, these parts of the distributions were not included in the minimization procedure. Overall, the features that were neglected in the minimization (apparent small particle distributions in Fig. 4 and large-diameter "tails") constitute less than $10 \%$ of the total volume.

The samples at 236.3 and $236.1 \mathrm{~K}$ in Fig. 4 were partially frozen, with maxima in the ice distributions at approximately 7.1 and $5.6 \mu \mathrm{m}$, respectively, while the sample at $234.9 \mathrm{~K}$ was completely frozen, with a maximum in the ice distribution at 

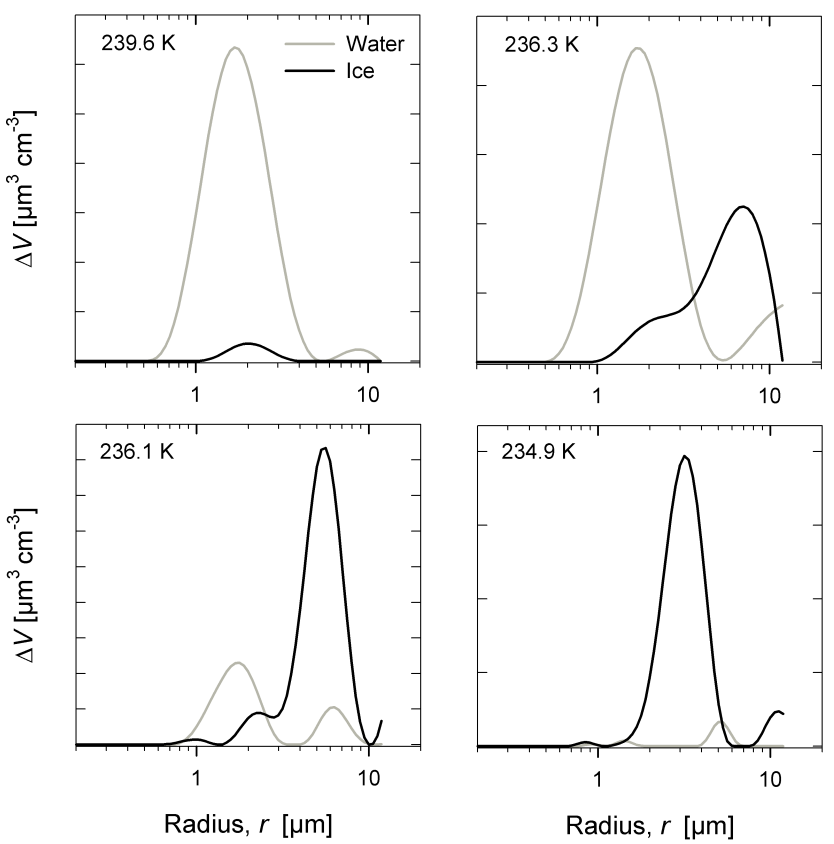

Fig. 4. Volume size distributions obtained using the aerosol retrieval procedure with the experimental spectra from freezing experiments using medium droplets in Fig. 3. The corresponding candidate freezing temperatures are indicated for the individual experiments.

about $3.2 \mu \mathrm{m}$. The residual water apparent in the distributions at $234.9 \mathrm{~K}$ is due to the similarity of refractive indices for supercooled water and ice considered above, and is neglected in the subsequent analysis.

The significant increases in the sizes of the ice particles relative to the initial supercooled water droplets are caused by deposition growth of the nucleated ice particles. The smaller size of the ice particles at lower temperature is a result of the temperature dependence of the growth process. Mass transfer is most significant near the nucleation point, where only a small fraction of the droplets have frozen and the saturation vapour pressure of water is high. As the temperature is reduced, the saturation vapour pressure of water decreases and a larger fraction of droplets freeze homogeneously, reducing the available liquid water for mass transfer and suppressing the growth of the ice particles.

It is important to emphasize that even for the completely frozen sample at $234.9 \mathrm{~K}$, ice nucleated in only a small fraction of the droplets, and these then grew significantly by mass transfer. Further decreases in the candidate freezing temperature to 233.5 and $232.4 \mathrm{~K}$ led to the nucleation of a larger fraction of the droplets, which did not grow as much as those at higher temperatures. The ice particle mean radii for these lower temperature cases (not shown) were 2.1 and $1.9 \mu \mathrm{m}$, respectively. At temperatures below $230 \mathrm{~K}$, the size distribution of the ice particles corresponds approximately to that of the initial size distribution of supercooled water,

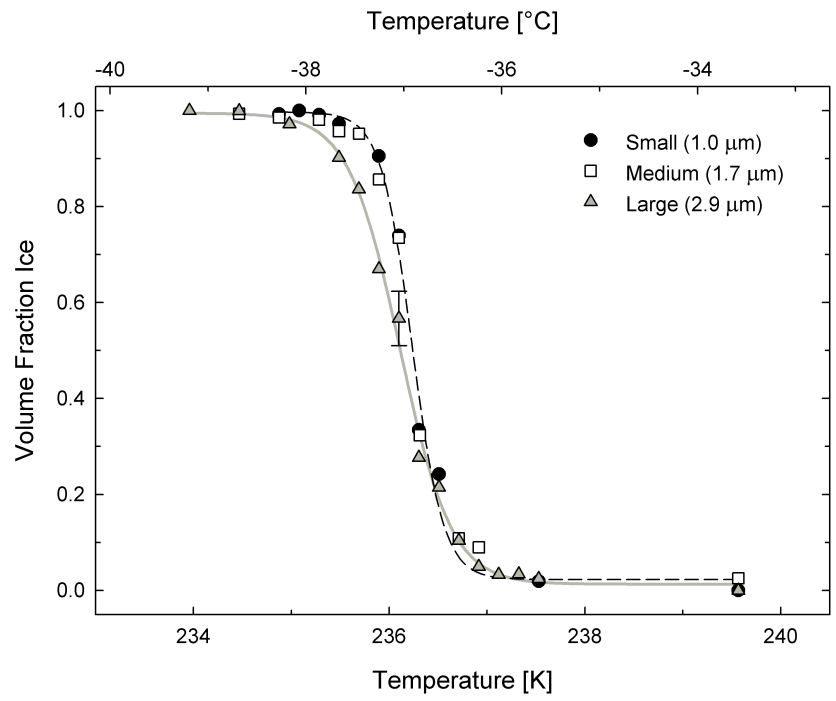

Fig. 5. Freezing curves determined from volume size distributions for experiments using each initial droplet size. Solid and dashed lines are provided to guide the eye and facilitate estimates of the homogeneous nucleation temperature from the freezing data.

indicating that homogeneous nucleation predominates and liquid-to-solid mass transfer growth is not significant. This is the expected consequence of the strong temperature dependences of both the nucleation rate and the mass transfer growth rate.

\subsection{Nucleation temperature determination from retrievals}

The volume size distributions of supercooled water and ice were used to calculate the volume fraction of ice - defined as the integrated volume concentration of ice particles divided by the sum of the integrated volume concentrations of ice and water particles - formed at each candidate freezing temperature. The resulting freezing curves for aerosol particles of each initial size are shown in Fig. 5. The curves for small and medium droplets overlap (dashed black line), and are shifted to slightly warmer temperatures than that for large droplets (solid grey line). We define the temperature at the mid-point of the curve, where the volume fraction of ice is 0.5 , to be the homogeneous nucleation point. For each particle size, the nucleation temperature is approximately $236 \mathrm{~K}$, in excellent agreement with values from previous work (Anderson et al., 1980; Cziczo and Abbatt, 1999; DeMott and Rogers, 1990). Errors in the volume fraction frozen range from 2 to $3 \%$ in the plateau regions, where the samples are predominantly supercooled droplets or ice, to $10 \%$ near the nucleation point, where the phase composition is most sensitive to small fluctuations in temperature. The upper limit for errors in the volume fraction of ice is illustrated by the error bar in Fig. 5, which is based on the larger uncertainty value near the nucleation point. 
The dependence of the volume fraction of ice on the candidate freezing temperature shows the evolution of the freezing process. However, the competition between homogeneous nucleation and mass transfer complicates the determination of the nucleation rate from these data, because the change in the volume of ice particles results from both processes. To isolate the contribution of homogeneous nucleation from that of mass transfer growth so that the nucleation rate coefficients could be calculated, it was necessary to use a microphysical model that contained both processes (see Sect. 3).

\subsection{Nucleation rate determination from model simulations}

Freezing experiments performed at different candidate freezing temperatures corresponded to different cross-sectional, mass-averaged temperature profiles obtained from a combination of direct measurements and high resolution CFD simulations (Sect. 2.4). Each experiment was interpreted in a separate model run. The input data for each run consisted of the measured initial and final supercooled water and/or ice aerosol particle distributions, the temperature profile, and the initial guesses for the parameters $A_{V}, B_{V}, \alpha_{\mathrm{w}}$ and $\alpha_{\text {ice. }}$. An example of the evolution of the volume distributions of supercooled water and ice that the model obtains for a freezing experiment performed at a candidate freezing temperature of 235.7 K is shown in Fig. 6 for medium-sized droplets generated by atomization. The corresponding temperature profile and evolution of the far-field pressure (Eq. 9), the number fraction of droplets nucleated, and volume fraction of ice (determined from the distributions) are given in Fig. 7.

The initial volume distribution of supercooled water droplets in Fig. 6 (solid black curve) has a maximum at a radius of $\sim 1.7 \mu \mathrm{m}$. As the aerosol travels to a region of lower temperature (see Fig. 7), the volume density of water droplets decreases while that of ice particles increases. Figure 6 shows that the ice particles become larger due to mass transfer following freezing; the mean radii are 2.9 and $3.7 \mu \mathrm{m}$ after residence times of 11.6 and $23.1 \mathrm{~s}$, respectively. The final sample, calculated for a $34.7 \mathrm{~s}$ residence time, is composed almost entirely of ice particles (blue dashed line; mean radius of $4.2 \mu \mathrm{m}$ ), with some residual water droplets.

The freezing experiment shown in Fig. 6 exemplifies a "partially frozen" case in which some liquid water droplets remain in the aerosol when it crosses the infrared detection beam. This corresponds to the vertical part of the freezing curve in Fig. 5, for which the volume fractions of ice are less than unity. Because water and ice are present simultaneously, these partially frozen experiments are preferable to cases where all of the water droplets freeze before detection in the lowermost section of the flow tube. In the absence of liquid water droplets, the kinetic timescale over which nucleation occurred is uncertain, and the mass accommodation coefficients required for the correct description of diffusion-limited mass transfer cannot be properly

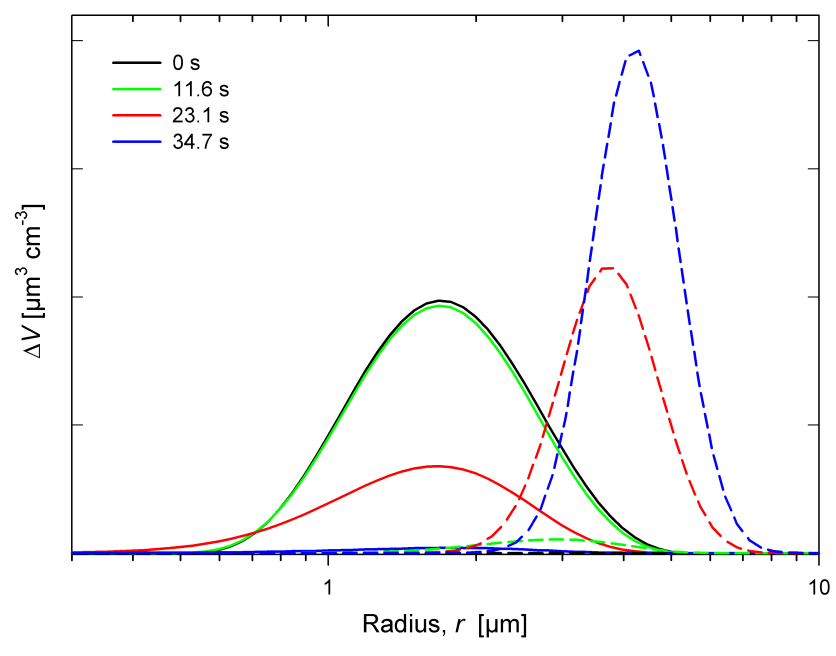

Fig. 6. Time evolution of volume distributions of supercooled water (solid lines) and ice (dashed lines) at 0,11.6, 23.1, and $34.7 \mathrm{~s}$ for a sample model run for medium droplets at $235.7 \mathrm{~K}$.
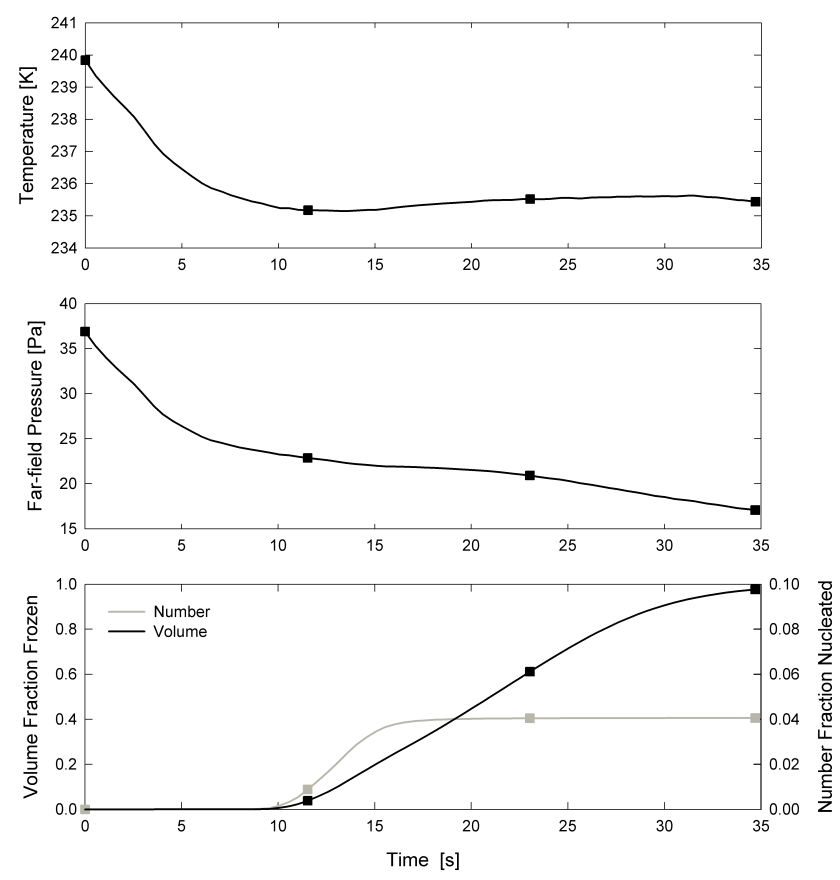

Fig. 7. Axial temperature profile and time evolution of far-field water vapour pressure, number fraction of droplets nucleated, and volume fraction of ice calculated by the model for the run at $235.7 \mathrm{~K}$ (medium droplets). The solid squares indicate the times for which the volume distributions of supercooled water and ice are shown in Fig. 6.

constrained. Accordingly, subsets of experiments with partially frozen aerosols for each initial droplet size were selected for subsequent model analysis: three experiments for small droplets at different candidate freezing temperatures, five experiments for medium droplets, and three experiments for large droplets. 


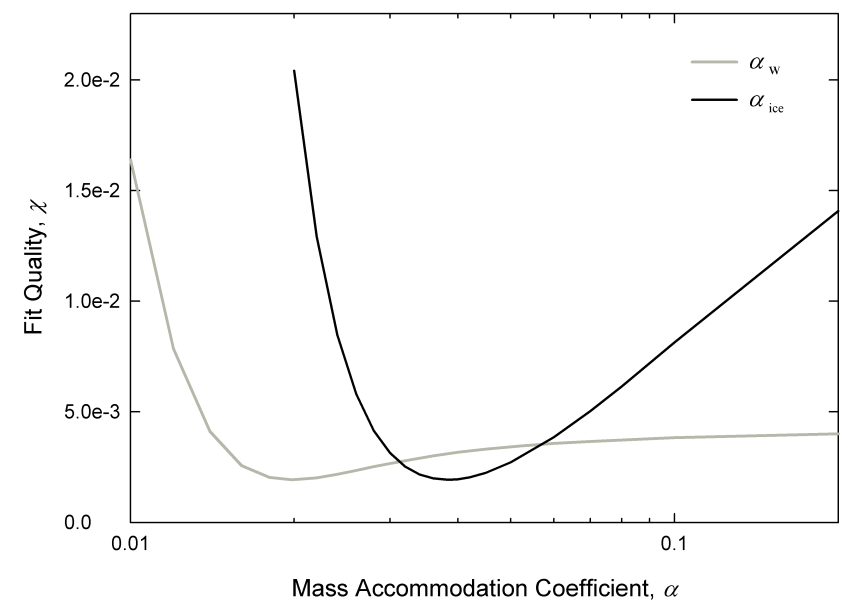

Fig. 8. Variation of $\chi$ with changes in mass accommodation coefficients $\alpha_{\mathrm{W}}$ and $\alpha_{\text {ice }}$ for the representative case of a partially frozen experiment for medium droplets at $235.7 \mathrm{~K}$.

Because ice particle growth by mass transfer is significant for partially frozen samples, the model runs corresponding to these experiments should be sensitive to the values of the mass accommodation coefficients $\alpha_{\mathrm{w}}$ and $\alpha_{\text {ice }}$, which determine the magnitudes of the gas-phase diffusion coefficients $D_{v}^{*}\left(r_{i}^{l}\right)$ and $D_{v}^{*}\left(r_{i}^{s}\right)$, respectively (see Sect. 4.1). To demonstrate this sensitivity, a series of model runs were performed in which the values of $\alpha_{\mathrm{w}}$ and $\alpha_{\text {ice }}$ were varied independently to find their influence on the value of $\chi$. The results for the experiment with partially frozen, medium-sized droplets at 235.7 $\mathrm{K}$ are shown in Fig. 8. In this case, the best fit between the measured and calculated ice and water size distributions (minimum value of $\chi$ ) was obtained with $\alpha_{\mathrm{w}}=0.020$ and $\alpha_{\text {ice }}=0.038$. The trends displayed in Fig. 8 are representative of those observed for aerosols with smaller and larger droplet sizes. The steeper minimum in $\chi$ as a function of $\alpha_{\text {ice }}$ indicates that the results are more sensitive to changes in the accommodation coefficient on ice than to changes in $\alpha_{\mathrm{w}}$. The variations in Fig. 8 show that these experiments can be used to estimate the mass accommodation coefficients - at least under our experimental conditions.

Fits to experimental distributions calculated from model runs for supercooled droplets with a mean radius of $1.7 \mu \mathrm{m}$ (medium-sized droplets) are shown in Fig. 9. The distributions producing the minimum value of $\chi$ are shown in each case. The associated values of $J_{V}$, calculated from the resulting values of $A_{V}$ and $B_{V}$, are plotted in Fig. 10.

As seen in the lower panel of Fig. 7, the largest number of droplets nucleates in a narrow range of temperatures $(\sim 0.25 \mathrm{~K})$ above the lowest temperature to which the particles are exposed in the AFT. This is due to the very strong temperature dependence of $J_{V}$, which changes by about two orders of magnitude per degree Kelvin (see Fig. 10). To obtain values of $J_{V}$ over as wide a temperature range as possi-
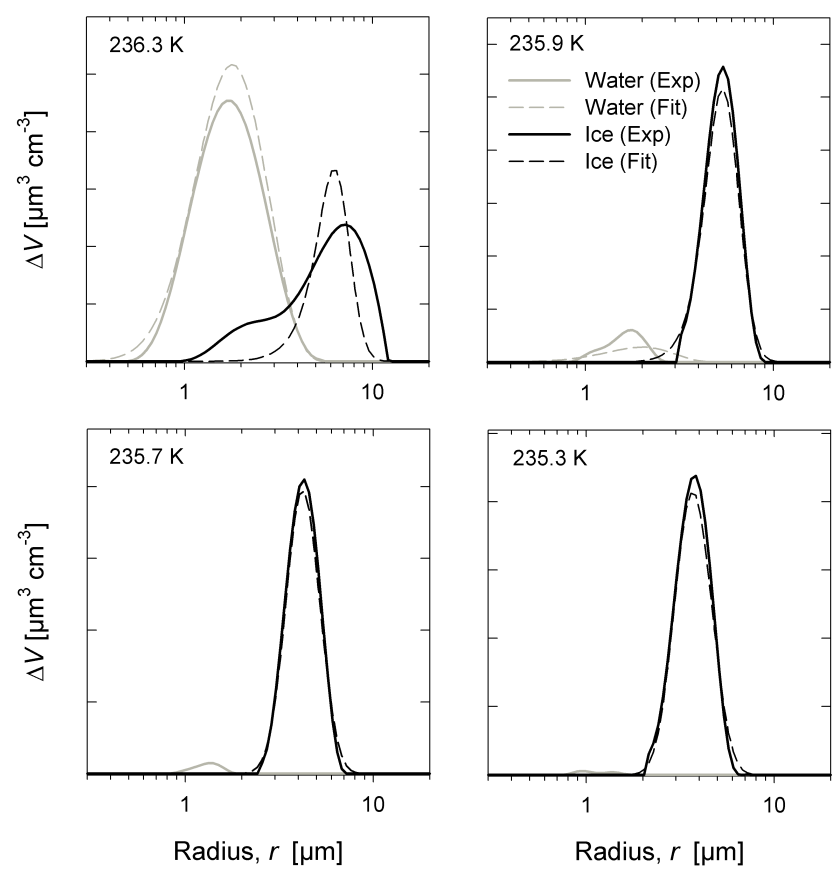

Fig. 9. Model fits to experimentally-measured distributions from partially frozen samples (medium droplets). The corresponding candidate freezing temperatures are indicated for the individual experiments.

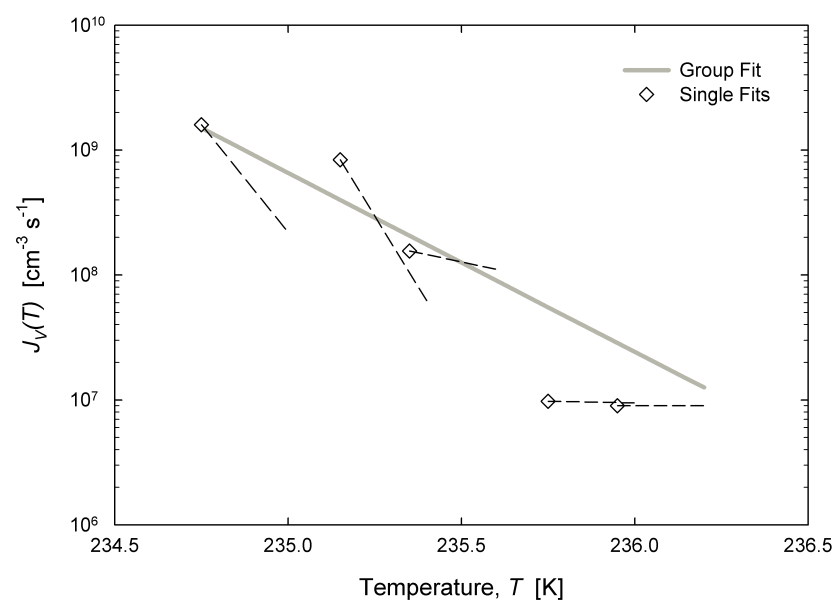

Fig. 10. Volume-based nucleation rate coefficients, $J_{V}$, determined from single and group fits to partially frozen experiments for medium droplets. The open diamonds indicate the lowest temperature reached in each freezing experiment, and the dashed lines show the temperature dependence of $J_{V}$ to $0.25 \mathrm{~K}$ above the minimum temperature.

ble and to mitigate the strong temperature dependence noted above, all five of the partially frozen experiments for medium droplets were fitted simultaneously, using a single set of minimization parameters. The result of this group fit is plotted as the solid curve in Fig. 10, showing the temperature dependence of the nucleation rate assuming that nucleation occurs 

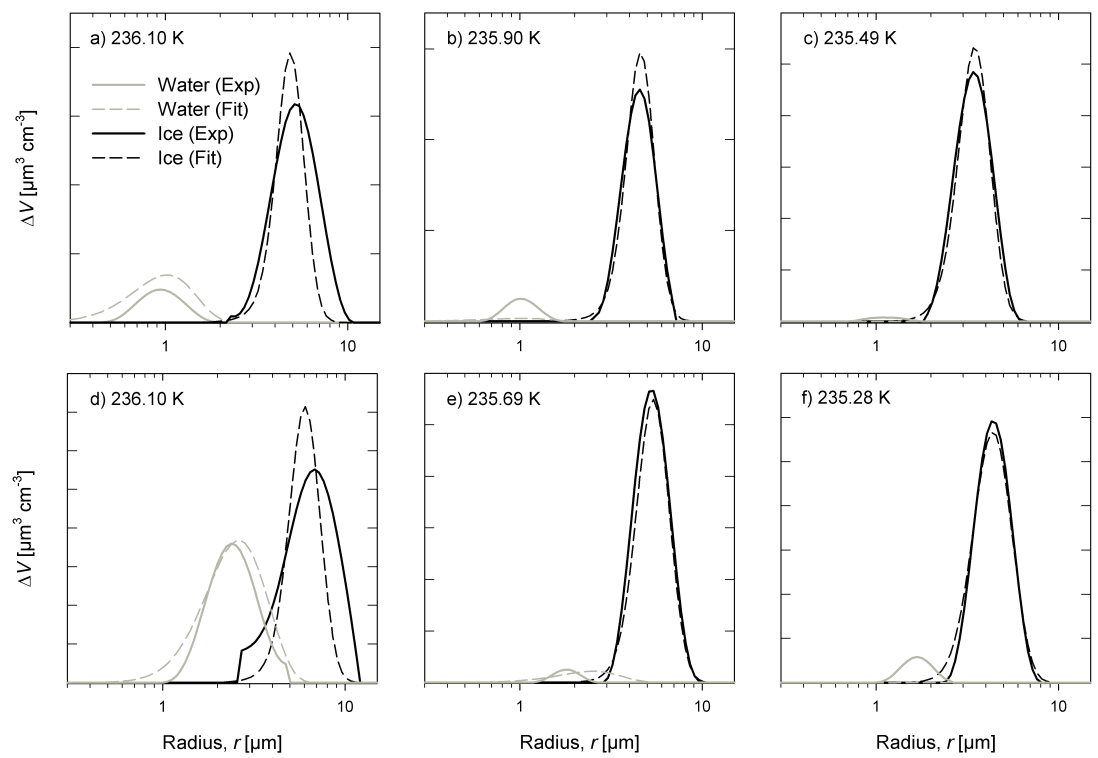

Fig. 11. Model results and comparison with experimentally measured size distributions for small and large droplets. The upper three panels (a) to (c) show results for the three experiments used in the small droplet group; the lower panels (d) to (f) the results of the large droplet group. The corresponding candidate freezing temperatures are indicated in the figure for the individual experiments.

within the droplet volume for samples in which most droplets have radii at or about $1.7 \mu \mathrm{m}$. Strictly speaking, this $J_{V}(T)$ curve applies only over the temperature range from $234.8 \mathrm{~K}$ to $236.2 \mathrm{~K}$; that is, from the minimum temperature in the experiment performed at the lowest AFT wall temperature of $235.3 \mathrm{~K}$ to $0.25 \mathrm{~K}$ warmer than the minimum temperature in the experiment conducted at the highest wall temperature of $236.5 \mathrm{~K}$ (see Fig. 10). It should be noted that the minima in the temperature profiles are slightly lower than the set-point (average) wall temperatures because of the cooling configuration, which is responsible for the $\pm 0.5 \mathrm{~K}$ axial gradient along tube sections.

Model fits to retrieved volume distributions from experiments with partially-frozen samples of small and large droplets, with mean radii of 1.0 and $2.9 \mu \mathrm{m}$, respectively, are shown in Fig. 11. The corresponding $J_{V}(T)$ curves obtained from group fits to these experiments are plotted with that determined from the group fit to medium droplet experiments in Fig. 12. The valid temperature ranges for $J_{V}(T)$ are 235.0 to $235.8 \mathrm{~K}$ for small droplets and 234.8 to $235.8 \mathrm{~K}$ for large droplets. Error bars at different temperatures are indicated for each group fit in Fig. 12. These reflect the influence of the strong temperature dependence of $J_{V}$ from individual freezing experiments on the values determined from group fits. They were determined by repeating the group fits for each droplet size $N$ times and removing one of the $N$ individual freezing experiments in each successive run. The standard deviations of the resulting $J_{V}(T)$ curves were used to determine the uncertainties shown for each droplet size.

The values of $\chi, A_{V}, B_{V}, \alpha_{\mathrm{w}}$ and $\alpha_{\text {ice }}$ obtained from the group fits for each droplet size are given in Table 1 . The val-

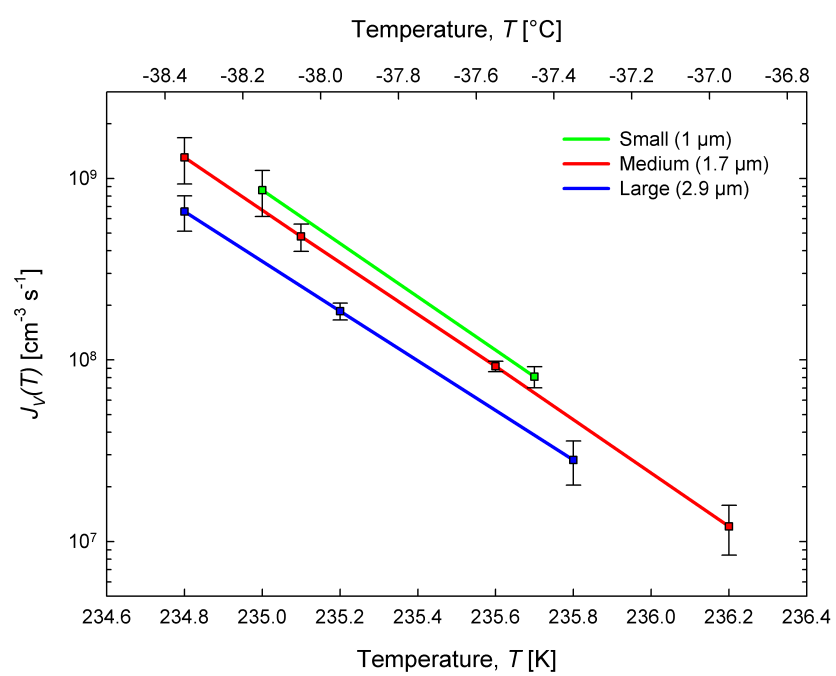

Fig. 12. Volume-based homogeneous nucleation rate coefficients, $J_{V}(T)$, determined from model fits to groups of experiments for small, medium, and large droplets.

ues of $A_{V}$ and $B_{V}$ can be used in Eq. (5) to calculate values of $J_{V}(T)$, the temperature-dependent volume nucleation rate coefficient, over the temperature range of our experiments. Averaging the compiled values of mass accommodation coefficients, weighted by the number of freezing experiments used in each group fit, results in $\alpha_{\mathrm{w}}=0.054 \pm 0.012$ and $\alpha_{\text {ice }}=0.031 \pm 0.001$. The uncertainties are estimated from the standard deviation of the values in Table 1 about the weighted averages. 
Table 1. Minimization parameters from group fits for each droplet size.

\begin{tabular}{cccccc}
\hline Radius $(\mu \mathrm{m})$ & $\chi$ & $A_{V}(J)$ & $B_{V}(J / K)$ & $\alpha_{\mathrm{w}}$ & $\alpha_{\text {ice }}$ \\
\hline 1.0 & $3.68 \times 10^{-3}$ & $-2.592393 \times 10^{-18}$ & $-1.186721 \times 10^{-20}$ & 0.054 & 0.030 \\
1.7 & $3.50 \times 10^{-3}$ & $-2.527704 \times 10^{-18}$ & $-1.159562 \times 10^{-20}$ & 0.063 & 0.030 \\
2.9 & $2.14 \times 10^{-3}$ & $-2.386266 \times 10^{-18}$ & $-1.100254 \times 10^{-20}$ & 0.039 & 0.032 \\
\hline
\end{tabular}

Diffusive mass transfer in the present study is based on the assumption that supercooled droplets and ice crystals have the same temperature as their surroundings; that is, that heat transfer due to evaporation from droplets or deposition on ice is negligible (see Sect. 3.1). This assumption can potentially bias the values of the nucleation rate and mass accommodation coefficients determined using the microphysics model. In a given freezing experiment, the number fraction of droplets nucleated is very small. For instance, Fig. 7 shows that approximately $4 \%$ of droplets froze in an experiment using medium droplets at $235.7 \mathrm{~K}$. Hence, there are many more droplets than ice particles. The rate of deposition on the ice particles far exceeds the rate of evaporation from the large number of droplets, and so the temperatures of individual droplets are not affected. At lower temperatures, the number fraction of droplets frozen increases; however, because the partial pressure of water vapour is correspondingly lower, the evaporation rate and hence the droplet cooling rate are both very small for this case as well.

Given the more rapid rates of deposition, the ice particle temperatures could be warmer than the surrounding gas. To investigate the influence of such temperature differences, a series of sensitivity tests were conducted using the microphysics model for the same groups of small, medium, and large droplet experiments discussed above. For temperature differences of up to $0.1 \mathrm{~K}$, which are an order of magnitude larger than the differences reported by Chelf and Martin (2001), the evaporation and deposition coefficients varied by less than $13 \%$ and $6 \%$, respectively, of their values assuming thermal equilibrium with the surrounding gas. For the same and larger (up to $0.3 \mathrm{~K}$ ) temperature differences, the nucleation rate coefficients varied within $4 \%$. The results of the sensitivity tests suggest that the negligible heat transfer approximation is valid for our experiments, and that changes in particle temperatures due to evaporation and/or deposition will not significantly influence our results.

The validity of our approach was assessed further by comparing model results with experimentally measured volume fraction frozen values (Sect. 4.2 and Fig. 5). For this test, the nucleation rate coefficients determined for each droplet size were used in a set of forward model simulations conducted over the temperature range of our experiments. In each simulation, the initial droplet size distribution was cooled according to a mass-averaged temperature profile computed from

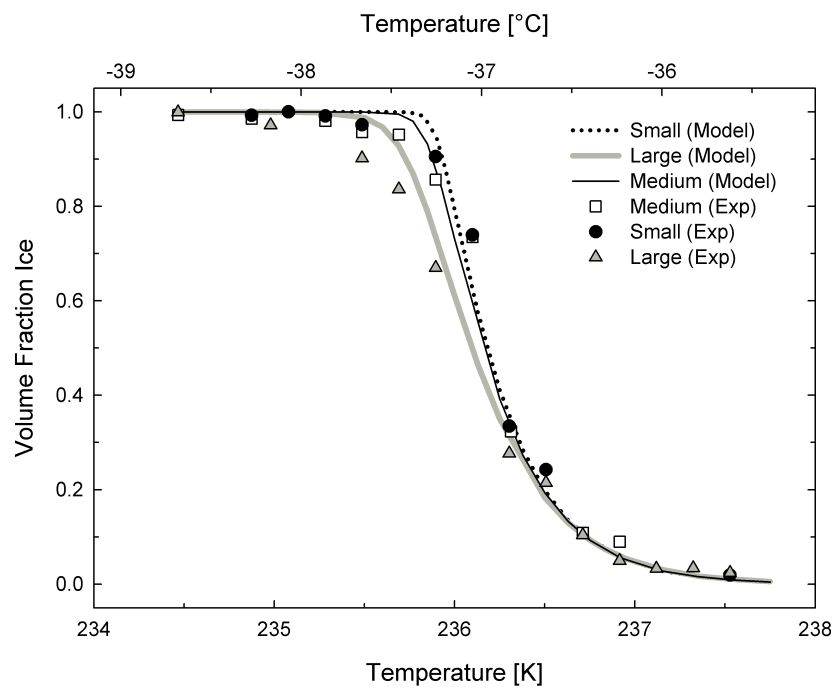

Fig. 13. Comparison of modelled volume fraction frozen curves for each droplet size with experimental (exp) values.

CFD (Sect. 2.4) for a particular candidate freezing temperature. The resulting supercooled water and/or ice size distributions were used to calculate the volume fraction frozen. The modelled values agree well with the experimental values and have similar size-dependences, as shown in Fig. 13. This agreement is an additional demonstration that the microphysical processes and temperature conditions in the freezing experiments are represented well by the model.

\subsection{Comparison of nucleation rate coefficients}

The nucleation rate coefficients determined in the present work are compared against values from previous studies of droplets created in an expansion chamber (DeMott and Rogers, 1990) and levitated in a cooled electrodynamic balance (Duft and Leisner, 2004; Krämer et al., 1999; Stöckel et al., 2005) in Fig. 14. The water droplets examined in the experiments of the other authors ranged in size from about 5 to $50 \mu \mathrm{m}$. The values of $J_{V}(T)$ determined in the present study agree best with those of Stöckel et al. (2005) in terms of both temperature dependence and magnitude, effectively extending their values to lower temperatures. The temperature dependence of our results is similar to those obtained by 


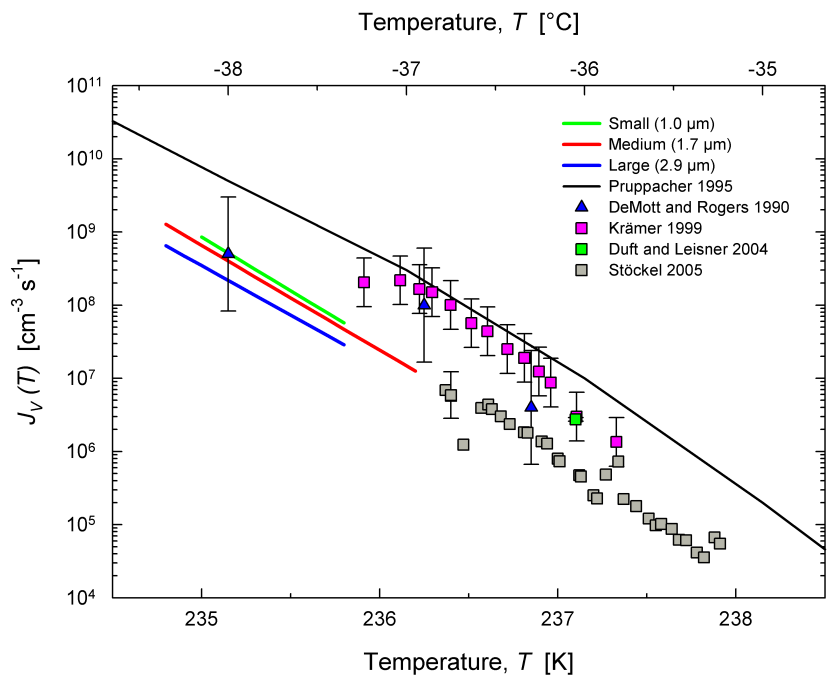

Fig. 14. Comparison of $J_{V}(T)$ curves determined from group fits in the present work with literature values determined using levitated droplets in electrodynamic balances (squares) and expansion cloud chambers (triangles). Error bars are provided for literature values only (uncertainties in the present study are indicated in Fig. 12).

Krämer et al. (1999) and Duft and Leisner (2004), as well as that of the curve of Pruppacher (1995), but the absolute values are lower. The Pruppacher recommendation is a fit to earlier experimental data, including those of DeMott and Rogers (1990), which agree with our values at low temperature, but have a different temperature dependence.

The droplet radii in each of the previous studies are larger than the $\sim 4 \mu \mathrm{m}$ threshold for surface nucleation proposed by Duft and Leisner (2004). The droplet size distributions in the present study, however, all have mean radii below this threshold, allowing one to consider whether classical nucleation theory is applicable to such droplets. In addition, all experiments in this study were performed in the same experimental apparatus under similar experimental conditions, allowing one to assess the size dependence of $J_{V}(T)$ with minimal contribution from systematic errors.

For a classical, volume-based freezing process, the nucleation rate depends on the droplet volume (function of $r^{3}$ ), and the rate coefficient $J_{V}(T)$ should be independent of droplet radius. This is not the case for the $J_{V}(T)$ curves for small, medium, and large droplets in the present study. It is evident from Figs. 12 and 14 that for these curves, the magnitude of $J_{V}(T)$ increases with decreasing droplet radius for a given temperature. The magnitude of this trend exceeds that of the experimental uncertainty shown by the error bars (Fig. 12) and also appears to scale with the change in particle size. This size dependence is consistent with what would be expected if surface nucleation contributes significantly to the total nucleation rate, as considered in Eq. (1). As the droplet size decreases, the surface-to-volume ratio increases, which would increase the contribution from $J_{S}$. These results suggest that classical nucleation theory does not adequately describe the freezing of small, micrometre-sized supercooled water droplets, which is consistent with previous considerations of a droplet size threshold for surface nucleation (Duft and Leisner, 2004).

\section{Conclusions}

The freezing of micrometre-sized water aerosol droplets with mean radii of $1.0,1.7$, and $2.9 \mu \mathrm{m}$ was studied as a function of temperature in a cryogenic AFT apparatus. Analysis of experimental extinction spectra using a previouslyreported characterization procedure provided the size distributions and phase composition of the aerosol samples. The temperature dependence of the volume fraction of ice formed in the samples indicated a homogeneous nucleation point of approximately $236 \mathrm{~K}$ for each droplet size, in good agreement with previous literature reports.

An aerosol microphysics model was used to determine values of the temperature-dependent, volume-based homogeneous nucleation rate coefficient, $J_{V}(T)$, from experimental measurements. Mass accommodation coefficients for water molecules on liquid water, $\alpha_{\mathrm{w}}$, and ice, $\alpha_{\mathrm{ice}}$, were obtained from the model fits to experimental measurements. The fits were particularly sensitive to the value of $\alpha_{\text {ice }}$, which is a key parameter in cloud models (e.g. Cotton et al., 2007; Jensen et al., 1998) because it influences the value of the vapourphase diffusion coefficient, and thus the rate of ice particle growth via mass transfer. Mass accommodation coefficients from model fits to groups of experiments for each droplet size were averaged to obtain $\alpha_{\mathrm{w}}=0.054 \pm 0.012$ and $\alpha_{\text {ice }}=0.031 \pm 0.001$.

The $J_{V}(T)$ curves determined for droplets with mean radii of 1.0,1.7, and $2.9 \mu \mathrm{m}$ show a distinct size dependence, with the magnitude of $J_{V}(T)$ increasing with decreasing droplet size. This indicates that the freezing of these droplets cannot be described solely using the classical, volume-based nucleation theory and that the contribution of surface nucleation appears to increase with decreasing droplet size, as would be expected from the higher surface-to-volume ratios. These results are consistent with the suggestion by Duft and Leisner (2004) that the surface nucleation rate could become comparable to the volume rate at radii below approximately $4 \mu \mathrm{m}$.

Surface nucleation of small droplets will modulate the formation of cirrus clouds, altering the concentrations and sizes of ice crystals that would be produced as compared to volume-only nucleation scenarios. This has potentially important ramifications for bulk parameterizations in numerical models, which typically employ temperature- and composition-dependent formulations for the homogeneous nucleation rate coefficient based solely on the classical, volume-based approach (Kärcher and Lohmann, 2002; Liu and Penner, 2005). Any contribution from a surface-based 
process will alter the predicted characteristics of ice formation and growth, and in turn, the predicted cloud microphysical and radiative properties. Further work is required to develop a size-dependent parameterization for homogeneous nucleation that accounts for both volume- and surface-based processes. This is considered in a related manuscript (Kuhn et al., 2009).

\section{Appendix A}

\section{Influence of radial temperature gradients}

The model detailed in Sect. 3 uses mass-averaged axial temperature profiles to describe the cooling of the particles, which dictates all concurrent microphysical processes. These profiles have been shown to represent the conditions in the flow very well (Khalizov et al., 2006a), but as noted previously in Sect. 2.4, they still might be subject to radial temperature gradients of less than $0.5 \mathrm{~K}$. Given the nonlinear nature of the freezing process, it is important to demonstrate that such gradients do not significantly influence the modeldetermined nucleation rate coefficients, which use massaveraged temperature profiles.

Radial temperature gradients would increase the range of particle residence times in the flow. Slightly colder temperatures at the tube wall and fins result in slower flow velocities and longer particle residence times, while the slightly warmer flow in the tube centre result in faster flow velocities and shorter particle residence times. To assess the effect of this range of particle residence times in our freezing experiments, 200 particle tracks distributed across the tube cross section were analyzed using CFD for a $10 \mathrm{~K}$ temperature drop in the flow tube (i.e. a candidate freezing temperature $10 \mathrm{~K}$ colder than the conditioning temperature). The tube geometry and boundary conditions for the simulations are described elsewhere (Khalizov et al., 2006a).

We first determined the total residence times of the particles in the entire flow section in order to classify the flow conditions. The CFD results indicated that approximately $50 \%$ of particles had residence times between 20 and $25 \mathrm{~s}$, while about $20 \%$ and $10 \%$ had residence times between 25 and $30 \mathrm{~s}$ and 30 and $35 \mathrm{~s}$, respectively. The remaining $20 \%$ had residence times longer than $35 \mathrm{~s}$. From this analysis, the flow cross section was divided into three "layers" - a core layer with the shortest residence times containing about 50\% of particles, a middle layer with intermediate residence times containing about $30 \%$ of particles, and an outside layer with the longest residence times containing about $20 \%$ of particles (Fig. A1). We then used this somewhat simplified classification to explore how non-uniform residence times and temperature profiles would influence the model results.

Separate axial temperature profiles were computed for each layer using CFD, following the same approach as in Sect. 2.4. The results showed that flow velocities of the

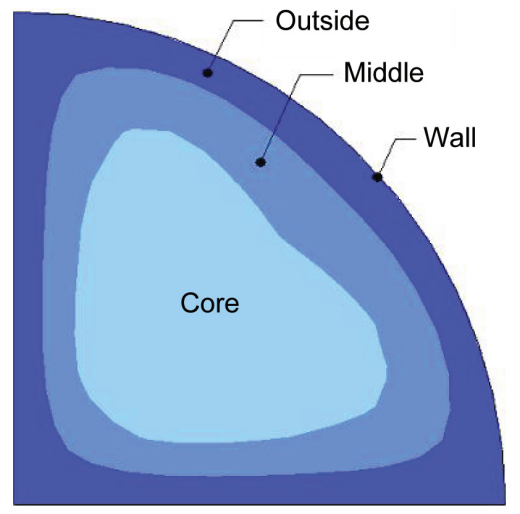

Fig. A1. Schematic representation of layers used in model simulations to assess the influence of radial temperature and velocity gradients in the flow tube.

aerosol in the core and outside layers were a factor of 1.5 and 0.7 of the velocity of the middle layer; that is, $50 \%$ faster and $30 \%$ slower, respectively, than the middle layer. Interactions between the layers by vapour diffusion were considered at each point along the axial temperature profile, and the mass accommodation coefficient values were fixed, at $\alpha_{\mathrm{w}}=0.054$ and $\alpha_{\text {ice }}=0.031$. These and previous CFD simulations showed that transport of particles across the layer boundaries was negligible. Each layer and axial profile used the same initial droplet size distribution (small, medium, or large droplets, as in Fig. 2). The final size distributions computed for each layer/profile were weighted according to the volume fraction of each layer intersected by the infrared beam in the observation section (approximately 0.25 for the core, 0.5 for the middle, and 0.25 for the outside layer) and averaged. The minimization was then done as in Sect. 3.2, except that it was based on the comparison between these new distributions and the experimental distributions.

The resulting $J_{V}(T)$ curves for group fits to small, medium, and large droplets are plotted in Fig. A2. The corresponding curves determined from model runs using mass-averaged temperature profiles - that is, the values from Figs. 12 and 13 - are shown for comparison. Clearly the $J_{V}(T)$ curves from the layered simulations are very close in magnitude to those from the mass-averaged temperature profiles and both results also have the same size dependences. In the averaged aerosol sample seen by the infrared beam, the shorter residence times in the core layer compensate for the longer residence times in the outside layer, and vice versa. Moreover, this analysis shows that the temperature profiles and flow velocities act to equalize the cooling rates in the different layers. The flow near the wall has a slower velocity but a more abrupt temperature decrease and vice versa for flow near the centre of the tube. This tendency to equalize the cooling rates across the radius also helps to minimize differences in this dimension. 


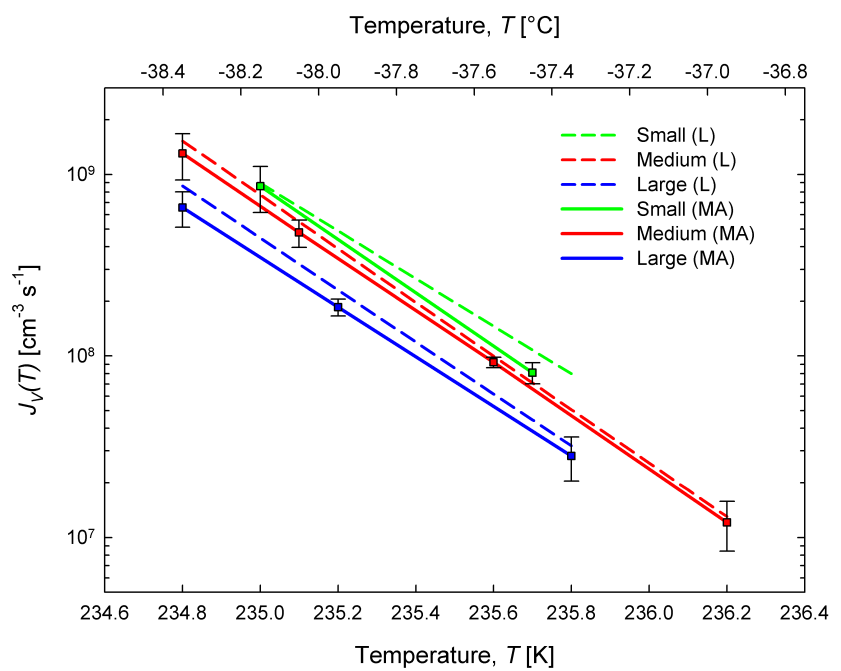

Fig. A2. Comparison of $J_{V}(T)$ curves from model simulations using layers $(\mathrm{L})$ to represent different temperature and velocity profiles in the flow tube with curves from simulations employing crosssectional and mass-averaged temperature profiles (MA).

Thus the results from the layered simulations confirm that the mass-averaged temperature profiles represent very well the conditions experienced by the particles in the flow tube, and show that the small residual temperature/velocity gradients effectively cancel each other out. Finally, it is important to keep in mind that our experiments employ a statistical ensemble of particles, and even if individual particles experience slightly different axial temperature profiles, this would not affect the validity of our results, which give the behaviour of the entire sample, rather than that of the individual particles.

Acknowledgements. This research was supported by the Natural Sciences and Engineering Research Council of Canada, the Canadian Foundation for Climate and Atmospheric Science, and the Ontario Research and Development Challenge Fund. The authors would like to thank Alex Zasetsky for invaluable input throughout this project, Brendan Pinto and Monica Harvey for assistance with the experimental measurements and microphysics model, and Wayde Johnson for conducting the CFD analysis of our AFT. We would also like to thank Dan Murphy and two anonymous reviewers for constructive comments that helped to improve this manuscript.

\section{Edited by: T. Koop}

\section{References}

Anderson, R. J., Miller, R. C., Kassner, J. L., and Hagen, D. E.: A study of homogeneous condensation-freezing nucleation of small water droplets in an expansion cloud chamber, J. Atmos. Sci., 37 , 2508-2520, 1980.

Bohren, C. F. and Huffman, D. R.: Absorption and scattering of light by small particles, John Wiley and Sons, Inc., New York, 530 pp., 1983.
Butorin, G. T. and Skripov, V. P.: Crystallization in supercooled water, Kristallografiya, 17, 379-0384, 1972.

Chelf, J. H. and Martin, S. T.: Homogeneous ice nucleation in aqueous ammonium sulfate aerosol particles, J. Geophys. Res., 106, 1215-1226, 2001.

Choularton, T. W. and Latham, J.: Measurements of deposition coefficient for ice, and its application to cirrus seeding, Q. J. Roy. Meteorol. Soc., 103, 307-318, 1977.

Clapp, M. L., Miller, R. E., and Worsnop, D. R.: Frequencydependent optical-constants of water ice obtained directly from aerosol extinction spectra, J. Phys. Chem., 99, 6317-6326, 1995.

Cotton, R. J., Benz, S., Field, P. R., Möhler, O., and Schnaiter, M.: Technical Note: A numerical test-bed for detailed ice nucleation studies in the AIDA cloud simulation chamber, Atmos. Chem. Phys., 7, 243-256, doi:10.5194/acp-7-243-2007, 2007.

Cziczo, D. J. and Abbatt, J. P. D.: Deliquescence, efflorescence, and supercooling of ammonium sulfate aerosols at low temperature: implications for cirrus cloud formation and aerosol phase in the atmosphere, J. Geophys. Res., 104, 13781-13790, 1999.

DeMott, P. J. and Rogers, D. C.: Freezing nucleation rates of dilutesolution droplets measured between $-30^{\circ} \mathrm{C}$ and $-40^{\circ} \mathrm{C}$ in laboratory simulations of natural clouds, J. Atmos. Sci., 47, 10561064, 1990.

Djikaev, Y. S., Tabazadeh, A., Hamill, P., and Reiss, H.: Thermodynamic conditions for the surface-stimulated crystallization of atmospheric droplets, J. Phys. Chem. A, 106, 10247-10253, 2002.

Duft, D. and Leisner, T.: Laboratory evidence for volumedominated nucleation of ice in supercooled water microdroplets, Atmos. Chem. Phys., 4, 1997-2000, doi:10.5194/acp-4-19972004, 2004.

Glickman, T.: Glossary of meteorology, American Meteorological Society, Boston, 855 pp., 2000.

Haynes, D. R., Tro, N. J., and George, S. M.: Condensation and evaporation of $\mathrm{H}_{2} \mathrm{O}$ on ice surfaces, J. Phys. Chem., 96, 85028509, 1992.

Heymsfield, A. J., Miloshevich, L. M., Schmitt, C., Bansemer, A., Twohy, C., Poellot, M. R., Fridlind, A., and Gerber, H.: Homogeneous ice nucleation in subtropical and tropical convection and its influence on cirrus anvil microphysics, J. Atmos. Sci., 62, 41-64, 2005.

Hinds, W. C.: Aerosol technology: properties, behavior, and measurement of airborne particles, Wiley-Interscience, Toronto, 483 pp., 1999.

Houzelot, J. L. and Villermaux, J.: Mass-transfer in annular cylindrical reactors in laminar-flow, Chem. Eng. Sci., 32, 1465-1470, 1977.

Hung, H. M., Malinowski, A., and Martin, S. T.: Ice nucleation kinetics of aerosols containing aqueous and solid ammonium sulfate particles, J. Phys. Chem. A, 106, 293-306, 2002.

Hung, H. M. and Martin, S. T.: Apparent freezing temperatures modeled for several experimental apparatus, J. Geophys. Res.Atmos., 106, 20379-20394, 2001.

Jensen, E. J., Toon, O. B., Tabazadeh, A., Sachse, G. W., Anderson, B. E., Chan, K. R., Twohy, C. W., Gandrud, B., Aulenbach, S. M., Heymsfield, A., Hallett, J., and Gary, B.: Ice nucleation processes in upper tropospheric wave-clouds observed during SUCCESS, Geophys. Res. Lett., 25, 1363-1366, 1998.

Kärcher, B. and Lohmann, U.: A parameterization of cirrus cloud formation: homogeneous freezing of supercooled aerosols, J. 
Geophys. Res.-Atmos., 107, 4010, doi:10.1029/2001JD000470, 2002.

Khalizov, A., Earle, M. E., Johnson, W. J. W., Stubley, G. D., and Sloan, J. J.: Development and characterization of a laminar aerosol flow tube, Rev. Sci. Instrum., 77, 033102, doi:10.1063/1.2175958, 2006a.

Khalizov, A. F., Earle, M. E., Johnson, W. J. W., Stubley, G. D., and Sloan, J. J.: Modeling of flow dynamics in laminar aerosol flow tubes, J. Aerosol. Sci., 37, 1174-1187, 2006b.

Krämer, B., Hubner, O., Vortisch, H., Woste, L., Leisner, T., Schwell, M., Ruhl, E., and Baumgartel, H.: Homogeneous nucleation rates of supercooled water measured in single levitated microdroplets, J. Chem. Phys., 111, 6521-6527, 1999.

Kuhn, T., Earle, M. E., Khalizov, A. F., and Sloan, J. J.: Size dependence of volume and surface nucleation rates for homogeneous freezing of supercooled water droplets, Atmos. Chem. Phys. Discuss., 9, 22929-22953, doi:10.5194/acpd-9-22929-2009, 2009.

Lagarias, J. C., Reeds, J. A., Wright, M. H., and Wright, P. E.: Convergence properties of the Nelder-Mead simplex method in low dimensions, Siam J. Optimiz., 9, 112-147, 1998.

Lawson, R. P., Baker, B. A., Pilson, B., and Mo, Q.: In situ observations of the microphysical properties of wave, cirrus, and anvil clouds - Part II: Cirrus clouds, J. Atmos. Sci., 63, 3186-3203, 2006.

Li, Y. Q., Davidovits, P., Shi, Q., Jayne, J. T., Kolb, C. E., and Worsnop, D. R.: Mass and thermal accommodation coefficients of $\mathrm{H}_{2} \mathrm{O}(\mathrm{g})$ on liquid water as a function of temperature, J. Phys. Chem. A, 105, 10627-10634, 2001.

Liou, K. N.: Influence of cirrus clouds on weather and climate processes: a global perspective, Mon. Weather Rev., 114, 11671199, 1986.

Liu, X. and Penner, J. E.: Ice nucleation parameterization for global models, Meteorol. Z., 14, 499-514, 2005.

Magee, N., Moyle, A. M., and Lamb, D.: Experimental determination of the deposition coefficient of small cirrus-like ice crystals near $-50{ }^{\circ} \mathrm{C}$, Geophys. Res. Lett., 33, L17813, doi:10.1029/2006GL026665, 2006.

Prakash, A., Bapat, A. P., and Zachariah, M. R.: A simple numerical algorithm and software for solution of nucleation, surface growth, and coagulation problems, Aerosol Sci. Tech., 37, 892898, 2003.
Pratte, P., Van Den Bergh, H., and Rossi, M. J.: The kinetics of $\mathrm{H}_{2} \mathrm{O}$ vapor condensation and evaporation on different types of ice in the range 130-210 K, J. Phys. Chem. A, 110, 3042-3058, 2006.

Pruppacher, H. R.: A new look at homogeneous ice nucleation in supercooled water drops, J. Atmos. Sci., 52, 1924-1933, 1995.

Pruppacher, H. R. and Klett, J. D.: Microphysics of clouds and precipitation, Kluwer Academic Publishers, Boston, 954 pp., 1998.

Shaw, R. A. and Lamb, D.: Experimental determination of the thermal accommodation and condensation coefficients of water, J. Chem. Phys., 111, 10659-10663, 1999.

Stöckel, P., Weidinger, I. M., Baumgartel, H., and Leisner, T.: Rates of homogeneous ice nucleation in levitated $\mathrm{H}_{2} \mathrm{O}$ and $\mathrm{D}_{2} \mathrm{O}$ droplets, J. Phys. Chem. A, 109, 2540-2546, 2005.

Tabazadeh, A., Djikaev, Y. S., Hamill, P., and Reiss, H.: Laboratory evidence for surface nucleation of solid polar stratospheric cloud particles, J. Phys. Chem. A, 106, 10238-10246, 2002 a.

Tabazadeh, A., Djikaev, Y. S., and Reiss, H.: Surface crystallization of supercooled water in clouds, P. Natl. Acad. Sci. USA, 99, 15873-15878, 2002b.

Taborek, P.: Nucleation in emulsified supercooled water, Phys. Rev. B, 32, 5902-5906, 1985.

Turnbull, J. and Fisher, J. C.: Rate of nucleation in condensed systems, J. Chem. Phys., 17, 71-73, 1949.

Villermaux, J.: Diffusion in a cylindrical reactor, Int. J. Heat Mass Trans., 14, 1963-1981, 1971.

Zasetsky, A. Y., Earle, M. E., Cosic, B., Schiwon, R., Grishin, I. A., McPhail, R., Pancescu, R. G., Najera, J., Khalizov, A. F., Cook, K. B., and Sloan, J. J.: Retrieval of aerosol physical and chemical properties from mid-infrared extinction spectra, J. Quant. Spectrosc. Radiat. Trans., 107, 294-305, 2007.

Zasetsky, A. Y., Khalizov, A. F., Earle, M. E., and Sloan, J. J.: Frequency dependent complex refractive indices of supercooled liquid water and ice determined from aerosol extinction spectra, J. Phys. Chem. A, 109, 2760-2764, 2005.

Zasetsky, A. Y., Khalizov, A. F., and Sloan, J. J.: Characterization of atmospheric aerosols from infrared measurements: simulations, testing, and applications, Appl. Opt., 43, 5503-5511, 2004a.

Zasetsky, A. Y., Khalizov, A. F., and Sloan, J. J.: Local order and dynamics in supercooled water: a study by IR spectroscopy and molecular dynamic simulations, J. Chem. Phys., 121, 69416947, 2004b. 